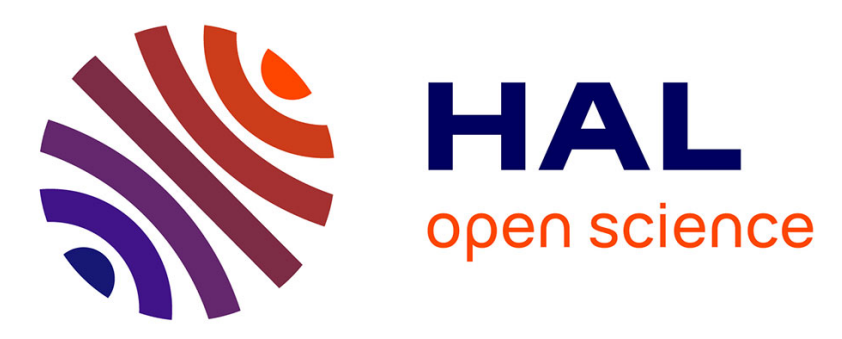

\title{
Period doubling bifurcation analysis and isolated sub-harmonic resonances in an oscillator with asymmetric clearances
}

Roberto Alcorta, Sébastien Baguet, Benoit Prabel, Philippe Piteau, Georges Jacquet-Richardet

\section{To cite this version:}

Roberto Alcorta, Sébastien Baguet, Benoit Prabel, Philippe Piteau, Georges Jacquet-Richardet. Period doubling bifurcation analysis and isolated sub-harmonic resonances in an oscillator with asymmetric clearances. Nonlinear Dynamics, 2019, 98, pp.2939-2960. 10.1007/s11071-019-05245-6 . hal02295420

\section{HAL Id: hal-02295420 \\ https://hal.science/hal-02295420}

Submitted on 24 Sep 2019

HAL is a multi-disciplinary open access archive for the deposit and dissemination of scientific research documents, whether they are published or not. The documents may come from teaching and research institutions in France or abroad, or from public or private research centers.
L'archive ouverte pluridisciplinaire HAL, est destinée au dépôt et à la diffusion de documents scientifiques de niveau recherche, publiés ou non, émanant des établissements d'enseignement et de recherche français ou étrangers, des laboratoires publics ou privés. 


\title{
Period doubling bifurcation analysis and isolated sub-harmonic resonances in an oscillator with asymmetric clearances
}

\author{
Roberto Alcorta (iD . Sebastien Baguet • Benoit Prabel · \\ Philippe Piteau · Georges Jacquet-Richardet
}

the date of receipt and acceptance should be inserted later

\begin{abstract}
In this paper, a frequency-domain characterisation of the period-doubling bifurcation is proposed. This allows an efficient detection and localisation of such points along frequency-response curves computed through continuation and the harmonic balance method. A simple strategy for branch switching to sub-harmonic regimes is presented as well. Furthermore, these bifurcations are tracked in a twodimensional parameter space, and extremum points with respect to the tracking parameter are characterized and linked to sub-harmonic isola formation. As a test case for these methods, a forced Duffing oscillator with asymmetric clearances is studied numerically. The results, which include the prediction of period-doubling cascades and sub-harmonic isolas, are then compared to experimental results, yielding an excellent agreement.
\end{abstract}

Keywords harmonic balance $\cdot$ bifurcation tracking period doubling $\cdot$ isola formation $\cdot$ clearance systems

\section{Introduction}

Strong nonlinearities, such as impacts or friction, give rise to complexity and multiple possible dynamic regimes, corresponding to specific zones in parameter space delimited by stability boundaries. A small variation in a given parameter, through which the system crosses one of these boundaries, may thus entail significantly different responses from an otherwise unchanged system. This corresponds to a bifurcation

R. Alcorta ( $ه) \cdot$ S. Baguet · G. Jacquet-Richardet

LaMCoS CNRS UMR5259, INSA-Lyon, Université de Lyon, 69621

Villeurbanne cedex, France

E-mail: roberto.alcorta@insa-lyon.fr

B. Prabel · P. Piteau

DEN-Service d'études mécaniques et thermiques (SEMT), CEA, Université Paris-Saclay,F-91191, Gif-Sur-Yvette, France event, whose prediction is of utmost importance in the context of engineering design and analysis.

An efficient way of directly determining stability boundaries is to employ continuation algorithms, which allow bifurcations to be tracked with respect to the system's parameters. This can be accomplished for bifurcations of a given type if a characterization is available, as is the case for all one and two-parameter-dependent bifurcations of cycles. The corresponding expressions represent constraints which, together with the equations of motion, constitute so-called extended systems. These can be found in textbooks on bifurcation theory, e.g. [1], and have been successfully incorporated into continuation codes such as AUTO [2], MATCONT [3] and COCO [4] for bifurcation analyses. It should be noted that all of the aforementioned codes rely on time-domain methods (namely, orthogonal collocation) to find periodic solutions to the non-linear equations of motion. An interesting alternative is to use frequency-domain methods such as the well-known Harmonic Balance Method (HBM), which are by their very nature well-suited to study periodic phenomena such as forced oscillations. This requires the equations characterizing bifurcations to be re-formulated in the frequency domain, but the principles of bifurcation tracking remain the same.

Xie et al. [5] characterized and tracked bifurcations of cycles from frequency-response curves by using pseudo arclength continuation on fully extended systems in the frequency domain. Minimally extended systems for the same purpose were proposed by Detroux et al [6]. Specifically, both of these works treated Limit Point (LP), Branch Point (BP) and Neimark-Sacker (NS) bifurcations of cycles, but not Period Doubling (PD), which is nonetheless a generic scenario that is common, amongst others, in systems with clearances [7], squeeze-film dampers [8], 1:2 internal resonances [9] or unilateral constraints [10, 11]. Also in [6], local extrema of LP bifurcation curves were found to in- 
dicate existence regions for detached resonances, or isolas. While the nonlinearity in that case was a piecewise-linear stiffness stemming from elastic contacts, a similar behaviour has also been observed in vibration absorbers [12] and beams attached to nonlinear springs [13], both of which include cubic restoring forces. As discussed by the authors of the latter paper, interactions between nonlinear modes lead to the birth of isolas in MDOF systems. Nonetheless, even SDOF systems have been reported to exhibit detached resonances in the presence of nonlinear damping $[14,15]$ or asymmetric clearances [16]. This last example is remarkable since, in contrast to all the other cited examples, the periodic cycles on the isolas were sub-harmonic: they responded at one half of the excitation frequency. As this is the main characteristic of oscillatory regim es past a PD bifurcation point, one could expect the formation of this particular kind of isola to be somehow related to period doubling. To the authors' best knowledge, however, no studies on the link between these two phenomena have been presented so far.

The purpose of the present work is twofold: firstly, an original frequency-domain extended system for calculating PD bifurcations in the framework of the HBM is proposed. Secondly, some local extrema of PD bifurcation curves are shown to be characteristic of sub-harmonic isola merging with the main response branch of forced vibrating systems. In addition, a test case consisting of a Duffing-like oscillator with asymmetric clearances is presented. Although the specific system under consideration was studied previously [17], the presence of sub-harmonic isolas was undetected before the present study. Furthermore, the analysis reveals that the degree of symmetry in the system is the fundamental parameter driving the behaviour of isolas for this case. This paper is organized as follows: Section 2 offers a quick review of the concepts associated with the solution of non-linear equations by the HBM, pseudo-arclength continuation and the notion of extended systems in bifurcation analysis. Section 3 presents the theoretical contributions of this work, which consist of an application of the previous techniques to the case of the period doubling bifurcation: detection, localisation and tracking. A simple method for switching to sub-harmonic branches is also discussed. Moreover, we propose an extended system for the detection and localisation of local extrema of bifurcation curves. The proposed methods are then applied to a test case, described in Section 4. The analysis, in Section 5, is divided into two parts: our new methods are first validated by comparison to the previously obtained results; next, new results made possible by bifurcation tracking are presented. We particularly discuss isolated sub-harmonic resonances, and proceed to show experimental measurements regarding this phenomenon. Finally, conclusions are drawn in Section 6.

\section{Numerical background}

\subsection{The Harmonic Balance Method}

We consider the generic vibration equations for a nonlinear system with $n$ degrees of freedom:

$\mathbf{M} \ddot{\mathbf{x}}(t)+\mathbf{C} \dot{\mathbf{x}}(t)+\mathbf{K x}(t)+\mathbf{f}_{\mathbf{N L}}(\mathbf{x}, \dot{\mathbf{x}})=\mathbf{p}(t)$

where $\mathbf{M}, \mathbf{C}$ and $\mathbf{K}$ are repectively the mass, damping and stiffness matrices of the underlying linear system, nonlinear forces are contained in the vector $\mathbf{f}_{\mathbf{n l}}(\mathbf{x}, \dot{\mathbf{x}})$ and $\mathbf{p}(t)$ is the external forcing. The main idea of the method is to look for a steady-state solution of Eq. (1) that is periodic, with minimal period $T=\frac{2 \pi}{\omega}$. Thus, all variables and force vectors are expanded as Fourier series up to the H-th harmonic:

$$
\begin{aligned}
\mathbf{x}(t) & =\left[\mathbb{T}(\omega t) \otimes \mathbf{I}_{n}\right] \mathbf{X} \\
\dot{\mathbf{x}}(t) & =\omega\left[\mathbb{T}(\omega t) \nabla \otimes \mathbf{I}_{n}\right] \mathbf{X} \\
\ddot{\mathbf{x}}(t) & =\omega^{2}\left[\mathbb{T}(\omega t) \nabla^{2} \otimes \mathbf{I}_{n}\right] \mathbf{X} \\
\mathbf{f}_{\mathrm{NL}}(\mathbf{x}, \dot{\mathbf{x}}) & =\left[\mathbb{T}(\omega t) \otimes \mathbf{I}_{n}\right] \mathbf{F}_{\mathrm{NL}}(\mathbf{X}) \\
\mathbf{p}(t) & =\left[\mathbb{T}(\omega t) \otimes \mathbf{I}_{n}\right] \mathbf{P} \\
\mathbb{T}(\omega t) & =[1, \cos (\omega t), \sin (\omega t), \ldots, \cos (H \omega t), \sin (H \omega t)]
\end{aligned}
$$

Here, $\otimes$ and $\nabla$ represent the Kronecker tensor product and the frequency-domain differential operator, respectively [5]. Uppercase vectors represent the Fourier coefficients of their lowercase counterparts. Autonomous systems $(\mathbf{p}(t)=\mathbf{0})$ have an undetermined phase due to the fact that they are invariant with respect to temporal translations; i.e., for any solution $\mathbf{x}(t)$ of (1), $\mathbf{x}\left(t+t_{0}\right)$ is also a solution for any $t_{0} \in \mathbb{R}$, which means that the Fourier coefficients are not uniquely determined. Thus, a particular solution must be fixed by imposing a socalled phase condition as an additional constraint and taking the oscillation frequency ${ }^{1}$ as an unknown. On the other hand, if $\mathbf{p}(t) \neq \mathbf{0}$, the phase is imposed by the forcing. Only the latter case is treated in what follows.

The transposition to the frequency domain is completed by a Galerkin projection of Eqs. (1) over the Fourier basis $\mathbb{T}(\omega t)$ through the inner product:

$\langle\mathbf{u}, \mathbf{v}\rangle=\frac{2}{T} \int_{0}^{T} \mathbf{u}^{*}(\tau) \cdot \mathbf{v}(\tau) d \tau$

where the symbol ${ }^{*}$ denotes the transpose of a vector. This operation is classical for Hilbert spaces, and in particular for the space of square-integrable functions on the interval $[0, T], L^{2}([0, T])$, where the solutions of (1) over one period are sought. Harmonic functions form an orthonormal basis, so that $\langle\mathbb{T}(\omega t), \mathbb{T}(\omega t)\rangle=\mathbf{I}_{2 H+1}$, and this projection yields

1 Throughout this paper, the term frequency will be systematically used to refer to an angular frequency in $\mathrm{rad} / \mathrm{s}$. 
the following set of $n(2 H+1)$ nonlinear algebraic equations for the Fourier coefficients $\mathbf{X}$ of the displacements:

$\mathbf{R}(\mathbf{X})=\mathbf{Z X}+\mathbf{F}_{\mathrm{NL}}(\mathbf{X})-\mathbf{P}=\mathbf{0}$

where the dynamic stiffness matrix is expressed as:

$\mathbf{Z}=\omega^{2} \nabla^{2} \otimes \mathbf{M}+\omega \nabla \otimes \mathbf{C}+\mathbf{I}_{2 H+1} \otimes \mathbf{K}$

Given an initial guess $\mathbf{X}_{0}$, Newton-Raphson iterations are used to find a solution of Eq. (4). Thus, at the k-th iteration:

$\mathbf{X}^{k+1}=\mathbf{X}^{k}-\left(\mathbf{R}_{\mathbf{X}}\left(\mathbf{X}^{k}\right)\right)^{-1} \mathbf{R}\left(\mathbf{X}^{k}\right)$

with the Jacobian:

$\mathbf{R}_{\mathbf{X}}=\mathbf{Z}+\frac{\mathbf{d F}_{\mathbf{N L}}}{\mathbf{d X}}$

These corrections are performed until a given accuracy is attained, for instance:

$\frac{\left\|\left(\mathbf{R}_{\mathbf{X}}\left(\mathbf{X}^{k}\right)\right)^{-1} \mathbf{R}\left(\mathbf{X}^{k}\right)\right\|}{\left\|\mathbf{X}^{k}\right\|}<\varepsilon, \varepsilon \in \mathbb{R}^{+}$

Nonlinear forces and their derivatives in the frequency domain are required for the computation of $\mathbf{F}_{\mathrm{NL}}$ and the Jacobian matrix $\mathbf{R}_{\mathbf{X}}$, respectively. These are generally difficult to obtain analytically for multiple harmonics and strong nonlinearities, but this is not the case for their time-domain counterparts, where a closed-form expression is usually available. As such, we apply the alternating frequency-time (AFT) technique [18]. This means that the Fourier coefficients $\mathbf{X}$ are first transformed to the time domain by an inverse FFT so that the derivatives can be evaluated analytically. Afterwards, a direct FFT is applied on the resulting signal to yield the frequency-domain derivatives.

Let us highlight two important features of this approach:

1. Certainly, the back-and-forth transformations are the most time-consuming part of the process. However, a high efficiency can be achieved by expressing the inverse and direct FFT as linear operators. The details can be found, for example, in [19].

2. Arbitrary nonlinearities may be treated in this manner without further work, which is convenient from an implementation viewpoint. This is true, in particular, for localised forces such as contacts, although a high number of harmonics and/or FFT sampling points may be required for the approximation to be accurate. Furthermore, note that Fourier basis functions are smooth (class $C^{\infty}$ ), hence some non-smooth non-linearities may pose convergence problems due to the Gibbs phenomenon [20]. However, piecewise continuous systems such as the one described in Section 4 represent a "mild" non-smoothness which can still be directly tackled by the HBM in some cases. An example of this was presented in [21]. Artificial regularization of non-smooth forces can, on the other hand, improve performance, at the cost of introducing an additional parameter. A discussion on different regularization strategies may be found in [22] and more recently in [23].

\subsection{Pseudo-arclength continuation}

A converged solution of Eq. (4) is associated to a set of fixed values of its parameters. Now, if one parameter is freed, this solution corresponds to a point on a curve in $(n(2 H+1)+1)$ dimensional space under the assumptions of the implicit function theorem [24]. Such a system is commonly said to have a codimension of 1 (denoted "codim-1"). By freeing $\omega$ and taking steps in codim-1 space, so-called frequency-response curves are obtained. Several different options exist concerning the way in which these steps are taken, the most popular ones being the Asymptotic Numerical Method [25] or the more traditional prediction-correction approaches, which include the well-known pseudo-arclength continuation method that we adopt herein.

Prediction A predicted solution is computed along the direction tangential to the curve at a given point $\left(\mathbf{X}_{p}, \omega_{p}\right)$ : $\mathbf{t}_{p}=[\Delta \mathbf{X} ; \Delta \omega]^{T}$. Since the system is underconstrained at this stage, an arclength equation is appended to provide closure:

$\|\Delta \mathbf{X}\|^{2}+\Delta \omega^{2}=\Delta s^{2}$

This equation fixes a step size in arclength coordinates. The predicted solution is given by:

$$
\left(\begin{array}{c}
\mathbf{X}_{p+1} \\
\omega_{p+1}
\end{array}\right)=\left(\begin{array}{c}
\mathbf{X}_{p} \\
\omega_{p}
\end{array}\right)+\left(\begin{array}{c}
\Delta \mathbf{X} \\
\Delta \omega
\end{array}\right)
$$

where:

$\left[\begin{array}{cc}\mathbf{R}_{\mathbf{X}} & \mathbf{R}_{\omega} \\ \Delta \mathbf{X}^{T} & \Delta \omega\end{array}\right]\left(\begin{array}{c}\Delta \mathbf{X} \\ \Delta \omega\end{array}\right)=\left(\begin{array}{c}\mathbf{0} \\ \Delta s^{2}\end{array}\right)$

Correction As the predicted solution is unlikely to lie on the response curve, Newton-Raphson corrections are made in a direction orthogonal to $\mathbf{t}_{p}$. At the $\mathrm{k}$-th iteration, the correction is computed as the solution to the system:

$$
\left[\begin{array}{cc}
\mathbf{R}_{\mathbf{X}}^{k} & \mathbf{R}_{\omega}^{k} \\
\Delta \mathbf{X}^{T} & \Delta \omega
\end{array}\right]\left(\begin{array}{c}
\delta \mathbf{X} \\
\delta \omega
\end{array}\right)=-\left(\begin{array}{c}
\mathbf{R}^{k} \\
0
\end{array}\right)
$$

where the superscript denotes evaluation at point $\left(\mathbf{X}_{p}^{k}, \omega_{p}^{k}\right)$. 


\subsection{Stability: Hill's method}

As the continuation procedure described in the last section computes both stable and unstable solutions, discrimination must be made between the two cases for each converged solution on the response curve. As an alternative to the usual time-domain method involving the monodromy matrix, stability can be evaluated directly in the frequency domain by applying Hill's method as proposed by [26]. It can be shown that this amounts to solving the quadratic eigenvalue problem:

$\left[\mathbf{R}_{\mathbf{X}}(\mathbf{X}, \omega)+\lambda_{i} \mathbf{D}_{1}(\omega)+\lambda_{i}^{2} \mathbf{D}_{2}\right] \phi_{i}=\mathbf{0}$

with:

$$
\left\{\begin{array}{c}
\mathbf{D}_{\mathbf{1}}(\omega)=2 \omega \nabla \otimes \mathbf{M}+\mathbf{I}_{2 H+1} \otimes \mathbf{C} \\
\mathbf{D}_{\mathbf{2}}=\mathbf{I}_{2 H+1} \otimes \mathbf{M}
\end{array}\right.
$$

Amongst the $2 n(2 H+1)$ solutions of (13), only $2 n$ actually correspond to the Floquet exponents of the system while the rest are a numerical artifice due to the multiplicity of harmonics, and provide redundant information. To extract the pertinent solutions, one can either sort eigenvalues or eigenvectors, the latter being seemingly a more robust approach [27]. A point $(\mathbf{X}, \omega)$ on the response curve corresponds to a stable periodic motion if the real parts of all its Floquet exponents is negative, and to an unstable one otherwise.

\subsection{Bifurcations in codimension- 1}

Changes of local (linearised) stability along solution branches indicate the appearance of new dynamical regimes, whose characteristics depend on the way that Floquet exponents $\lambda_{i}$ cross the imaginary axis under parameter variation. Three different scenarios may arise:

1. $\operatorname{Im}\left(\lambda_{i}\right)=0 \Longrightarrow$ static bifurcation: limit point (generic case) or branch point (degenerate case)

2. $\left.\operatorname{Im}\left(\lambda_{i, j}\right)= \pm m, m \in\right] 0, \frac{\omega}{2}[\Longrightarrow$ Neimark-Sacker bifurcation: appearance of a branch of quasi-periodic solutions.

3. $\operatorname{Im}\left(\lambda_{i, j}\right)= \pm \frac{\omega}{2} \Longrightarrow$ period doubling bifurcation: appearance of a branch of double-period solutions.

In the context of numerical continuation, it is usual to define test functions whose zeros correspond to bifurcation points, see [28] for examples. If a sign change occurs between two consecutive steps, a bifurcation is detected and can then be precisely localized by iteratively solving an appropriate extended system composed of the dynamical equilibrium equations (1) and additional equations characterizing a particular bifurcation. For instance, an extended system for LP calculation is:

$\mathbf{Y}_{\mathrm{LP}}(\mathbf{X}, \phi, \omega)=\left(\begin{array}{c}\mathbf{R}(\mathbf{X}, \omega) \\ \mathbf{R}_{\mathbf{X}}(\mathbf{X}, \omega) \phi \\ \phi^{*} \phi-1\end{array}\right)$
It can be seen that the eigenvector $\phi$ becomes an additional unknown and that both a normalization and the singularity of the Jacobian are imposed, thus yielding a closed system. A bifurcation point corresponds to $\left(\mathbf{X}_{\mathbf{0}}, \phi_{\mathbf{0}}, \omega_{0}\right)$ such that $\mathbf{Y}_{\mathrm{LP}}\left(\mathbf{X}_{\mathbf{0}}, \phi_{\mathbf{0}}, \omega_{0}\right)=\mathbf{0}$.

\section{Period doubling}

\subsection{Characterization}

As stated in Section 2.4, the condition to be fulfilled is to have a unique pair of purely imaginary Floquet exponents with magnitude $\lambda_{i, j}= \pm i \frac{\omega}{2}$, while all other exponents have negative real parts. Replacing for $\lambda_{i}$ in (13), writing the complex eigenvector as $\phi=\phi_{R}+i \phi_{I}$ and separating real and imaginary parts, we obtain the following set of equations:

$$
\left[\begin{array}{cc}
\mathbf{R}_{\mathbf{X}}-\left(\frac{\omega}{2}\right)^{2} \mathbf{D}_{\mathbf{2}} & \frac{\omega}{2} \mathbf{D}_{\mathbf{1}} \\
-\frac{\omega}{2} \mathbf{D}_{\mathbf{1}} & \mathbf{R}_{\mathbf{X}}-\left(\frac{\omega}{2}\right)^{2} \mathbf{D}_{\mathbf{2}}
\end{array}\right]\left(\begin{array}{c}
\phi_{\mathbf{R}} \\
\phi_{\mathbf{I}}
\end{array}\right)=\left(\begin{array}{c}
\mathbf{0} \\
\mathbf{0}
\end{array}\right)
$$

Notice that, by appending (16) to the equilibrium equations (4), we are left with $3 n(2 H+1)$ equations for $3 n(2 H+1)+1$ unknowns. Closure comes from a normalization equation for the eigenvector $\phi$. This can be done in a number of ways, such as imposing $g\left(\phi_{\mathbf{R}}, \phi_{\mathbf{I}}\right)=\|\phi\|-1=0$. However, in this paper we choose:

$g\left(\phi_{\mathbf{R}}, \phi_{\mathbf{I}}\right)=\mathbf{q}^{*} \cdot \phi_{\mathbf{I}}-b=0$

where $b \in[0,1]$ and $\mathbf{q}$ is a vector with non-zero projection over $\operatorname{Span}\left(\phi_{\mathbf{R}}, \phi_{\mathbf{I}}\right)$. In practice, the actual choice for $\mathbf{q}$ is unimportant, but it is recommended to take a vector with unit norm for numerical convenience. For instance, one could choose $\mathbf{q}=\mathbf{e}_{i}$, a unit vector, thus fixing the magnitude of the $\mathrm{i}$-th component of $\phi_{\mathbf{I}}$. Since the eigenvector is defined up to a multiplicative constant and, given Eq. (16), a non-trivial $\phi_{\mathbf{R}}$ is associated to any non-zero vector $\phi_{\mathbf{I}}$, this is a reasonable choice.

Hence, our new extended system is:

$\mathbf{Y}_{\mathrm{PD}}\left(\mathbf{X}, \phi_{\mathbf{R}}, \phi_{\mathbf{I}}, \omega\right)=\left(\begin{array}{c}\mathbf{R} \\ \left(\mathbf{R}_{\mathbf{X}}-\left(\frac{\omega}{2}\right)^{2} \mathbf{D}_{\mathbf{2}}\right) \phi_{\mathbf{R}}+\frac{\omega}{2} \mathbf{D}_{\mathbf{1}} \phi_{\mathbf{I}} \\ \left.\mathbf{R}_{\mathbf{X}}-\left(\frac{\omega}{2}\right)^{2} \mathbf{D}_{\mathbf{2}}\right) \phi_{\mathbf{I}}-\frac{\omega}{2} \mathbf{D}_{\mathbf{1}} \phi_{\mathbf{R}} \\ g\left(\phi_{\mathbf{R}}, \phi_{\mathbf{I}}\right)\end{array}\right)$

Letting $\mathbf{U}=\left(\mathbf{X}, \phi_{\mathbf{R}}, \phi_{\mathbf{I}}, \omega\right)$, we have at the k-th solver iteration:

$\mathbf{U}^{k+1}=\mathbf{U}^{k}-\left[\mathbf{J}_{\mathbf{A}}\left(\mathbf{U}^{k}\right)\right]^{-1} \mathbf{Y}_{\mathrm{PD}}\left(\mathbf{U}^{k}\right)$ 
with the extended Jacobian given by:

$\mathbf{J}_{\mathbf{A}}(\mathbf{U})=\left[\begin{array}{cccc}\mathbf{R}_{\mathbf{X}} & \mathbf{0}_{\mathbf{L}} & \mathbf{0}_{\mathbf{L}} & \mathbf{R}_{\omega} \\ \left(\mathbf{R}_{\mathbf{X}} \phi_{\mathbf{R}}\right)_{\mathbf{X}} & \mathbf{R}_{\mathbf{D}} & \frac{\omega}{2} \mathbf{D}_{\mathbf{1}} & \widetilde{\mathbf{R}}_{1} \\ \left(\mathbf{R}_{\mathbf{X}} \phi_{\mathbf{I}}\right)_{\mathbf{X}} & -\frac{\omega}{2} \mathbf{D}_{\mathbf{1}} & \mathbf{R}_{\mathbf{D}} & \widetilde{\mathbf{R}}_{2} \\ \mathbf{0}^{*} & \mathbf{0}^{*} & \mathbf{p}^{*} & 0\end{array}\right]$

and:

$$
\left\{\begin{array}{c}
\mathbf{R}_{\mathbf{D}}=\mathbf{R}_{\mathbf{X}}-\left(\frac{\omega}{2}\right)^{2} \mathbf{D}_{\mathbf{2}} \\
\widetilde{\mathbf{R}}_{1}=\left(\mathbf{R}_{\mathbf{X}} \phi_{\mathbf{R}}\right)_{\omega}-\frac{\omega}{2} \mathbf{D}_{\mathbf{2}} \phi_{\mathbf{R}}+\widehat{\mathbf{D}}_{1} \phi_{\mathbf{I}} \\
\widetilde{\mathbf{R}}_{2}=\left(\mathbf{R}_{\mathbf{X}} \phi_{\mathbf{I}}\right)_{\omega}-\frac{\omega}{2} \mathbf{D}_{\mathbf{2}} \phi_{\mathbf{I}}-\widehat{\mathbf{D}}_{1} \phi_{\mathbf{R}} \\
\widehat{\mathbf{D}}_{1}(\omega)=2 \omega \nabla \otimes \mathbf{M}+\frac{1}{2} \mathbf{I}_{L} \otimes \mathbf{C}
\end{array}\right.
$$

The computation of the second-derivative terms defined in (21) is once again achieved through the AFT procedure. Details on this can be found, for instance, in [5]. As usual, the equality $\mathbf{Y}_{\mathrm{PD}}\left(\mathbf{U}_{\mathbf{0}}\right)=\mathbf{0}$ is not enforced but rather the iterations are stopped when a certain $\mathbf{U}_{\mathbf{0}}$ satisfies a criterion similar to (8):

$$
\frac{\left\|\left(\mathbf{J}_{\mathbf{A}}\left(\mathbf{U}^{k}\right)\right)^{-1} \mathbf{Y}_{P D}\left(\mathbf{U}^{k}\right)\right\|}{\left\|\mathbf{U}^{k}\right\|}<\boldsymbol{\varepsilon}, \boldsymbol{\varepsilon} \in \mathbb{R}^{+}
$$

\subsection{Detection: Test function}

We propose two different test functions for detecting period doubling bifurcations.

First, notice that the matrix on the left-hand side of (16), which is called $\mathbf{B}$ hereafter, is singular for a solution of the extended system (18). One could then be tempted to use its determinant as a test function $\varphi_{P D}=\operatorname{det}(\mathbf{B})$, but this is not an appropriate choice since this function is positive-definite ${ }^{2}$. However, for this same reason, bifurcation point is a local minimum for the determinant, i.e. the derivative of the determinant changes sign at such a point. Thus, one can use a finite-difference approximation to the derivative and define:

$\varphi_{\mathrm{PD}, \mathrm{p}}^{1}=\operatorname{sign}\left(\operatorname{det}\left(\mathbf{B}_{\mathrm{p}}\right)-\operatorname{det}\left(\mathbf{B}_{\mathrm{p}-1}\right)\right)$

Then, a period doubling bifurcation is detected between two consecutive continuation steps $p$ and $p+1$ if: $\varphi_{\mathrm{PD}, \mathrm{p}}^{1} \cdot \varphi_{\mathrm{PD}, \mathrm{p}+1}^{1}<0$.

While this function accomplishes its purpose, it is somewhat inconvenient due to the fact that it may not be used at the very first continuation step. Alternatively, note that the Floquet exponents are available from Hill's method. Thus, by letting:

$\bar{\lambda}=\max _{j=\{1, \ldots, 2 n\}}\left\|e^{\frac{2 \pi \lambda_{j}}{\omega}}\right\|$

\footnotetext{
2 In the sense that, following a coordinate transform which places the bifurcation point at the origin, $f(\mathbf{0})=0$ and $f(\mathbf{x})>0 \forall \mathbf{x} \neq 0$.
}

a simple test function is given by:

$\varphi_{\mathrm{PD}}^{2}=\operatorname{sign}(\operatorname{Re}(\bar{\lambda}))|\bar{\lambda}|+1$

and, similarly, $\varphi_{\mathrm{PD}, \mathrm{p}}^{2} \cdot \varphi_{\mathrm{PD}, \mathrm{p}+1}^{2}<0$ implies the presence of a bifurcation between steps $p$ and $p+1$. Since no additional determinant calculations are required, this latter test function is more efficient than the previous one.

The two points between which a bifurcation has been detected represent suitable starting guesses for the iterations (19), but performance may be enhanced by selecting the one with the smaller magnitude of the test function.

\subsection{Branching}

Recall that the Fourier basis $\mathbb{T}(\omega t)$ from (2) includes only terms whose frequencies are multiples of the fundamental frequency $\omega$. This means, first of all, that sub-harmonic responses can not be accurately computed with this basis alone, but also that period-doubling bifurcations can be readily identified as such. Indeed, consider the alternative basis:

$\mathbb{T}_{s}^{H}(\omega t)=\left[1, \cos \left(\frac{\omega}{2} t\right), \sin \left(\frac{\omega}{2} t\right), \cos (\omega t), \sin (\omega t), \ldots\right]$

Since $\mathbb{T}^{H}(\omega t) \subset \mathbb{T}_{s}^{H}(\omega t)$, any T-periodic solution to the equations of motion can be constructed in this new basis. However, the addition of sub-harmonic functions means that the minimal period is now $2 T$. As a consequence, the period doubling bifurcation is not associated with a change in period any more: instead, it identifies a symmetry-breaking phenomenon, which corresponds to a degenerate static bifurcation, or branch point. This is no novelty, as various authors [1] have recognized the relation between this two types of bifurcations in terms of the underlying Poincare maps of dynamical systems. The operation $\omega \leftarrow \frac{\omega}{2}$ which transforms $\mathbb{T}(\omega t)$ into $\mathbb{T}_{s}(\omega t)$ is equivalent to considering the second-iterate application of the original system, with the corresponding Poincaré map.

By grouping the sub-harmonic sine and cosine functions in:

$$
\begin{aligned}
\mathbb{T}_{\frac{1}{2}}^{H}(\omega t)= & {\left[\cos \left(\frac{\omega}{2} t\right), \sin \left(\frac{\omega}{2} t\right), \ldots,\right.} \\
& \left.\cos \left(\frac{(2 H+1) \omega}{2} t\right), \sin \left(\frac{(2 H+1) \omega}{2} t\right)\right]
\end{aligned}
$$

it is easily be seen that $\mathbb{T}_{s}^{H}(\omega t)=\mathbb{T}^{H}(\omega t) \cup \mathbb{T}_{\frac{1}{2}}^{H}(\omega t)$ and that these two sets are mutually orthogonal through the inner product (3), as illustrated in Fig. 1. Geometrically, this means that we can construct a vector tangent to the sub-harmonic branch by simply taking the tangent vector from the most recent predictor step and adding a component belonging to the subspace generated by $\mathbb{T}_{\frac{1}{2}}^{H}$ :

$\mathbf{t}_{\mathrm{PD}}=\mathbf{t}_{\mathrm{p}}+\delta \mathbf{t}_{\frac{1}{2}}$ 


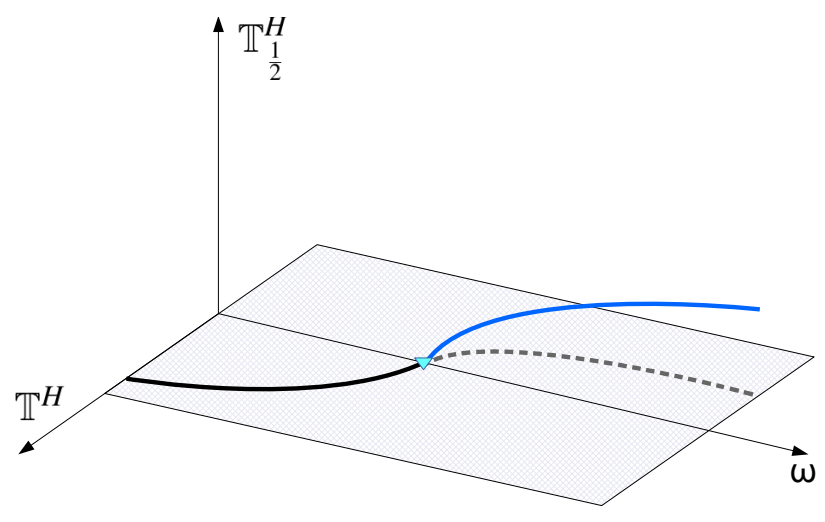

Fig. 1: Branching onto a sub-harmonic branch.

Determining the vector $\mathbf{t}_{\mathrm{PD}}$ is straightforward by applying the well-known Algebraic Branching Equation (ABE) [29] to the system expressed on the $\mathbb{T}_{s}^{H}(\omega t)$ basis; details on this are given in Appendix A.

The following steps summarize the procedure for subharmonic branching:

1. Localize a period-doubling bifurcation by the techniques from Sections 3.1 and 3.2.

2. Include sub-harmonics, i.e. change the original Fourier basis from $\mathbb{T}^{H}$ to $\mathbb{T}_{s}^{H}$.

3 . Use the $A B E$ to find a tangent vector $\mathbf{t}_{\mathrm{PD}}$ along the subharmonic branch.

4. Take a predictor step in the direction given by $\mathbf{t}_{\mathrm{PD}}$.

5. Apply corrections by orthogonal Newton-like iterations until convergence to a solution of (4).

It should be noted that, while following a sub-harmonic branch, further period doubling bifurcations may be encountered. This may indicate the presence of a sub-harmonic cascade, one of the best known routes to chaos [30]. If this is the case, one can follow the cascade up to arbitrary orders by repeatedly applying the procedure described above. However, the result of directly doubling the number of subharmonics at each new bifurcation is a large system with mostly null terms. A (sub-) harmonic selection procedure similar to those proposed by [31], for example, is useful to avoid this unnecessary numerical burden.

\subsection{Tracking}

Consider a parameter $\gamma$ of system (1) which was kept fixed at a value $\gamma_{0}$ up to this point. By the Implicit Function Theorem, the point $\left(\mathbf{U}_{\mathbf{0}}, \gamma_{0}\right)$ corresponding to a period doubling bifurcation belongs to a curve in a codimension- 2 vector space. As before, we can fully determine this bifurcation curve by pseudo-arclength continuation, with $\gamma$ now included amongst the unknowns of the problem. This means that the extended Jacobian is now given by:

$\mathbf{J}_{\mathbf{A A}}=\left[\begin{array}{cc}\mathbf{J}_{\mathbf{A}} & \frac{\partial\left(\mathbf{Y}_{\mathrm{PD}}\right)}{\partial \gamma} \\ \Delta \tilde{\mathbf{U}}^{*} & \Delta \gamma\end{array}\right]$

where:

$\Delta \tilde{\mathbf{U}}=\left(\Delta \mathbf{X}^{*}, \mathbf{0}^{*}, \mathbf{0}^{*}, \Delta \omega\right)^{*}$

When projected on the $(\omega, \gamma)$-plane, the curves obtained in this manner define stability boundaries across which the system shows quantitatively different dynamical behaviours.

\subsection{Local extrema in codimension-2}

While performing bifurcation tracking, a constraint equation fixes some of the eigenvalues of the system's Jacobian matrix. For instance, in the case of a period doubling curve, two eigenvalues are imposed to be purely imaginary and to have a magnitude of $\frac{\omega}{2}$ on every point. However, all the remaining eigenvalues can vary as a function of the bifurcation parameters $(\omega$ and a second parameter $\gamma)$. As a result, it is possible that another eigenvalue reaches a critical value such as 0 or $\left.\pm \kappa i, \kappa \in] 0, \frac{\omega}{2}\right]$ for some point $\left(\omega_{0}, \gamma_{0}\right)$, referred to as a codimension-2 bifurcation. Hereafter we focus on the case of an additional zero eigenvalue appearing along a bifurcation curve. By analogy with the codim-1 case, this can be called a codim-2 LP and corresponds geometrically to a turning point, thus to a local extremum with respect to the tracking parameter.

Such a point is of great practical interest due to its role as an organizing centre for the dynamics. As shown schematically in Fig. 2, the local extremum marks the limiting value of $\gamma$ beyond which multi-stability occurs for certain ranges of $\omega$.

It should be noted that, actually, limit points themselves correspond to local extrema with respect to $\omega$ in codim-1 space, since:

$\frac{d \mathbf{X}}{d \omega}=-\left(\mathbf{R}_{\mathbf{X}}\right)^{-1} \mathbf{R}_{\omega}$

is undetermined due to the singularity of the Jacobian, which indicates a vertical tangent in $(\mathbf{X}, \omega)$ coordinates ${ }^{3}$. In the same way, generic local extrema are nothing but limit points of the bifurcation curve with respect to $\gamma$. This viewpoint is convenient from a computational perspective, since it allows us to re-use the typical extended system for limit points (15) to detect, localise and eventually track local extrema. As

\footnotetext{
3 Note that a vertical tangent may also occur in the case of a pitchfork bifurcation, see Appendix A. However, assuming that the singularity indeed corresponds to an LP, the geometrical argument presented here holds true.
} 


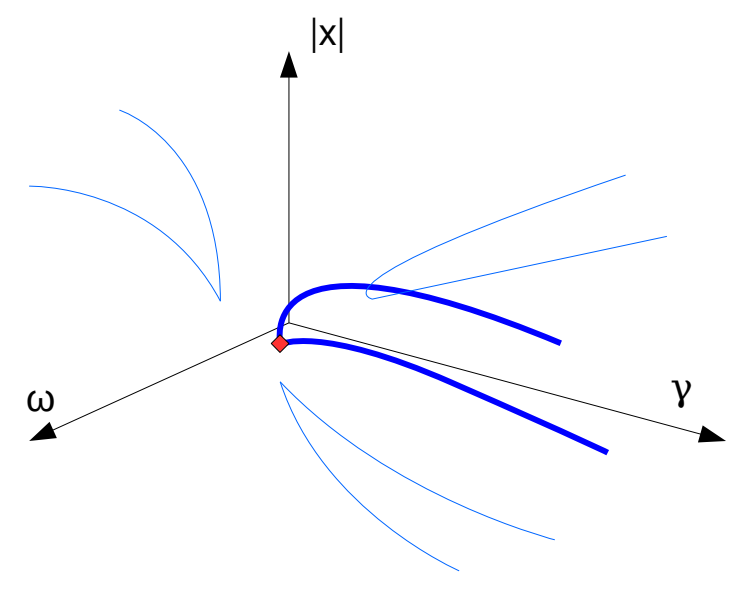

Fig. 2: Schematic representation of a codim-2 bifurcation: $(\omega, \gamma)$ are parameters, $|\mathbf{x}|$ is a given measure for displacement.

before, let $\mathbf{U}=(\mathbf{X}, \phi, \omega)$ and consider a generic extended system $\mathbf{Y}^{\text {bif }}$ defining a codimension-1 bifurcation being tracked with respect to $\gamma$. A local extremum of the bifurcation curve is a point $(\mathbf{U}, \gamma)$ which is a root of the new extended system defined by:

$\mathbf{W}_{\mathrm{ex}}^{\mathrm{bif}}(\mathbf{U}, \Psi, \gamma)=\left(\begin{array}{c}\mathbf{Y}^{\mathrm{bif}} \\ \mathbf{Y}_{\mathbf{U}}^{\mathrm{bif}} \Psi \\ \Psi^{*} \Psi-1\end{array}\right)$

where $\Psi$ is the eigenvector associated to the zero eigenvalue of the extended Jacobian $\mathbf{Y}_{\mathbf{U}}^{\text {bif }}$. Note that one might use:

$\varphi_{\mathrm{ex}, \mathrm{p}}=\operatorname{det}\left(\mathbf{Y}_{\mathbf{U}, \mathrm{p}}^{\mathrm{bif}}\right)$

as a test function. Likewise, a local extremum may be detected by a change of sign of $\Delta \gamma$, and thus a simple alternative is to use:

$\varphi_{\mathrm{ex}, \mathrm{p}}=\Delta \gamma_{p}$

In what follows, we will focus on the case of period-doubling curves: $\mathbf{Y}^{\text {bif }}:=\mathbf{Y}_{\mathrm{PD}}$. Thus, we may re-write (32) as:

$\mathbf{W}_{\mathrm{ex}}^{\mathrm{PD}}(\mathbf{U}, \Psi, \gamma)=\left(\begin{array}{c}\mathbf{Y}_{\mathrm{PD}} \\ \mathbf{J}_{\mathbf{A}} \Psi \\ \Psi^{*} \Psi-1\end{array}\right)$

The Jacobian matrix associated with this extended system has indeed the same form as the one for a classical limit point:

$\mathbf{J}_{A}^{W}=\left[\begin{array}{ccc}\mathbf{J}_{\mathbf{A}} & \mathbf{0}_{L} & \frac{\partial\left(\mathbf{Y}_{\mathrm{PD}}\right)}{\partial \gamma} \\ \frac{\partial\left(\mathbf{J}_{\mathbf{A}} \Psi\right)}{\partial \mathbf{U}} & \mathbf{J}_{\mathbf{A}} & \frac{\partial\left(\mathbf{J}_{\mathbf{A}} \Psi\right)}{\partial \gamma} \\ \mathbf{0}^{*} & 2 \Psi^{*} & 0\end{array}\right]$
Letting $\mathbf{V}=(\mathbf{U}, \Psi, \gamma)$, the bifurcation may be approximated through Newton-like iterations:

$\mathbf{V}^{k+1}=\mathbf{V}^{k}-\left(\mathbf{J}_{\mathbf{A}}^{\mathbf{W}}\left(\mathbf{V}^{k}\right)\right)^{-1} \mathbf{W}_{\mathrm{ex}}^{\mathrm{PD}}\left(\mathbf{V}^{k}\right)$

Afterwards, it is straightforward to go one step further and use continuation on the extended system (35) to track these bifurcations in a codimension- 3 space, but this is not considered in this paper.

\subsection{Sub-harmonic isolas}

Recently, LP tracking has been found to reveal the presence of isolas in forced, nonlinear mechanical systems. As seen e.g. in [6] and [13], extrema in the LP curves indicate the formation of isolas at low forcing and their merging with the resonance peak of the main response curve at high forcing. Between these two points, isolas can be found. Moreover, since the LPs on the isolas and on the main response curve lie on the same bifurcation curve, it follows that the cycles on both of them are qualitatively similar. In particular, this means that the frequency of isolated solutions corresponds to the excitation frequency, as is the case for resonant solutions. Thus, if sub-harmonic or other kinds of isolas are present, they will not be detected by directly applying this method.

We now discuss a mechanism of sub-harmonic isola formation. A non-degenerate local extremum of the PD curve as discussed here is indeed an instance of the generalized period doubling bifurcation as defined and investigated in [32]. Interestingly, besides the collision of the two PD branches, the theory also predicts the emergence of a limit point curve at this point, as shown schematically in Fig. 3. This can be understood easily in terms of eigenvalues: a zero eigenvalue of the system's Jacobian exists at a local extremum of the PD curve, and so this point also belongs to an LP curve, which is entirely composed of solutions with a zero eigenvalue. However, the saddle cycles which make up this LP curve are necessarily sub-harmonic, implying the existence of a pair of 2T-periodic cycles (one stable, the other unstable) in the immediate vicinity of the bifurcation. In the context of forced responses of mechanical systems, it is clear that these cycles cannot be part of the main response branch, which is composed of T-periodic solutions. The only option left for them is then to form isolas, i.e., closed loops of solutions detached from the main branch. Moreover, since having a single point of change of stability in a closed loop is impossible, a second LP must necessarily exist on the isola. Thus, one can hypothesize that further varying the parameter which caused detachment will result in these saddle cycles coming closer together until they finally meet and the isolated curve collapses to a codimension- 2 point beyond which no isola exists at all. Such a point is then an isola centre, correspond- 


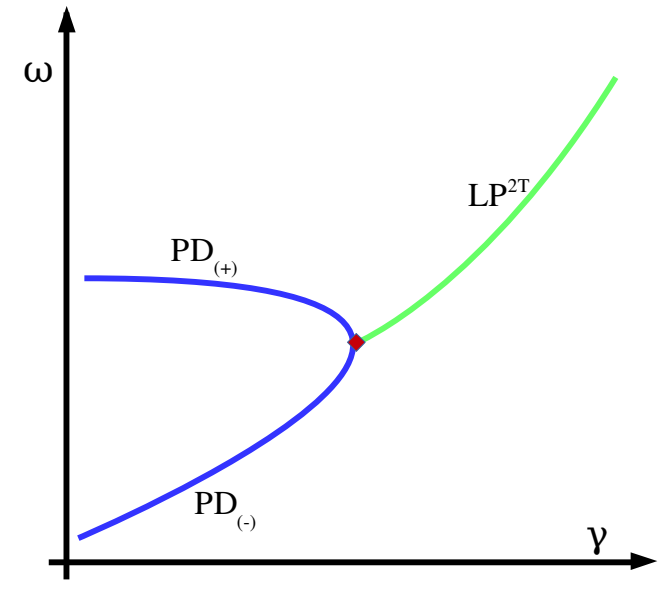

Fig. 3: Local bifurcation diagram near a Generalized Period Doubling point.

ing to the birth of a sub-harmonic attractor ${ }^{4}$. The practical steps for localizing a sub-harmonic isola centre can then be expressed as follows:

1. Detect and localize a PD bifurcation on the main response curve, as per Sects. 3.1 and 3.2.

2. Track this bifurcation with respect to a parameter $\gamma$, as per Sect. 3.4.

3. Detect and localize a generalized period doubling point on the PD curve, as per Sec. 3.5.

4. Change to sub-harmonic basis (Sect. 3.3) and use this point to launch the tracking of LP bifurcations with respect to $\gamma$.

5. Detect and localize a local extremum on the LP curve.

To conclude this section, let us remark that local extrema have long been recognized as being characteristic of isola formation in a general sense. Indeed, Dellwo et al. [33] explore this idea and use a perturbation method to solve for isola centres, with an application to chemical reactors. Later on, Kernevez et al. [34] applied continuation of isola formation points with a design perspective on a similar chemical system with the help of the software AUTO. Both of these papers formulate extended systems equivalent to (32), but differ in their approach to tackle the problem. The present contribution offers yet another option, this time in the framework of frequency-domain methods.

\footnotetext{
${ }^{4}$ As before, note that we can talk about supercritical as well as subcritical isola centres, corresponding to the "birth" and "death" of isolas, respectively.
}

\section{Test case}

\subsection{System description}

In this section, the techniques and concepts discussed previously are applied to an example system, shown in Fig. 4. The apparatus in this photograph was first studied by de Langre et al. in [17]. It was conceived as a simple mechanism which exhibits chaos, with the purpose of validating time integration algorithms. In that paper, it was observed that both measured and calculated chaotic regimes had a rich sub-harmonic spectrum, i.e., frequencies below that of the external forcing, and thus a sub-harmonic cascade was suggested as the likely route to chaos. However, a detailed bifurcation analysis was deemed out of the scope of their study. This is, in turn, the objective of the remainder of this paper.

\subsection{Modelling}

Fig. 5(a) shows a schematic representation of our system. It consists of a heavy concrete block supported by two clamped, slender steel bars on its sides. A mono-harmonic external excitation $p(t)=p \cos (\omega t)$ is provided by a fixed electromagnet whose oscillating magnetic field drives a coil, attached to the main block, sinusoidally. In this configuration, the system is constrained to move in only one direction. Displacement amplitude, denoted by $x(t)$, is measured relative to the rest position of the block's centre of mass, which coincides with the location of a rigid stop. One elastic spring lies on each side of the stop, so that the mass undergoes intermittent contacts when the displacement amplitude is greater in magnitude than at least one of the gaps, which are adjustable and allowed to be asymmetrical: contacts happen for $x(t)>j_{2}$ or $x(t)<-j_{1}$. In this study, we limit ourselves to the clearancetype system, where both $j_{1}$ and $j_{2}$ are positive, as opposed to the pre-loaded type. The springs are chosen to have a stiffness $K_{c}$ which is larger than the stiffness $k$ of the linear system's first bending mode. As explained in [17], the geometrical and material parameters were carefully chosen so that the system's first natural frequencies were far apart on the spectrum. Indeed, for the first bending mode, $f_{1}=5 \mathrm{~Hz}$ and $f_{3}=80 \mathrm{~Hz}$ for the third mode, whereas the second one has no contribution on the motion of the mass. Thus, if the frequencies associated to both the external forcing and the contact stiffness are kept low enough we can consider the motion to be largely dominated by the first mode. The limited parameter ranges used later in this paper are chosen to verify this constraint. As a consequence, the system is modelled by a forced SDOF oscillator of the form:

$m \ddot{x}(t)+c \dot{x}(t)+k x(t)+f_{\mathrm{NL}}(x)=p \cos (\omega t)$ 


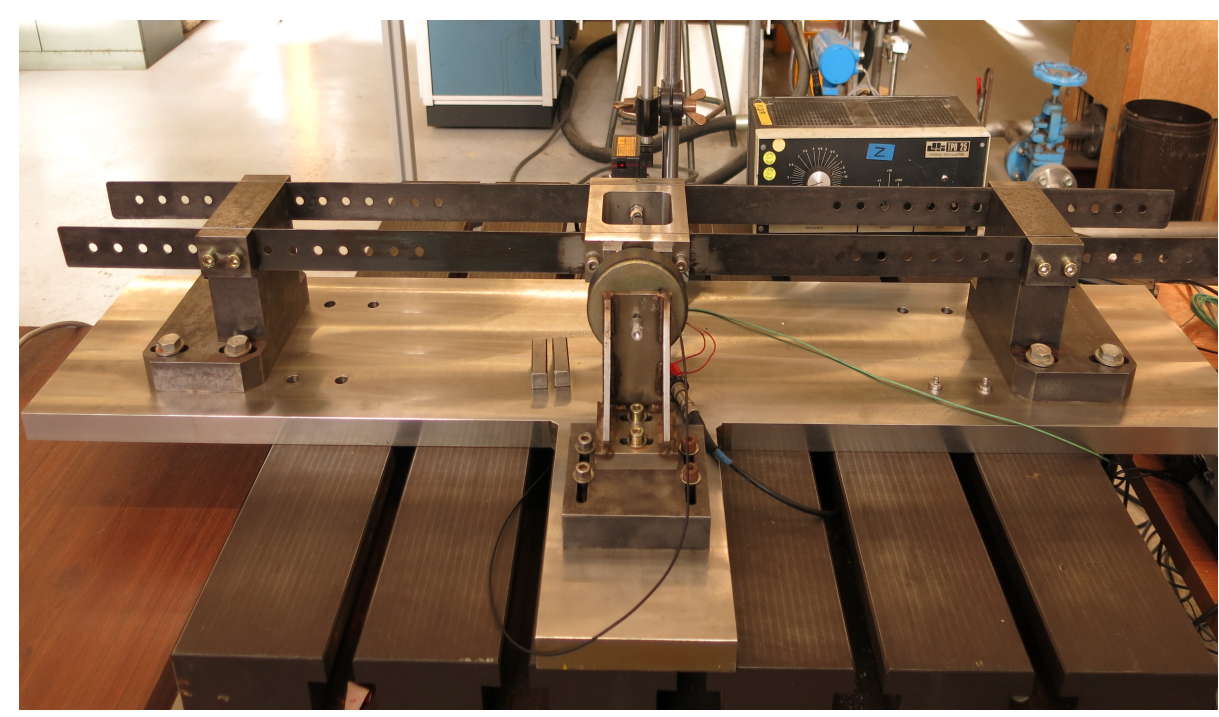

Fig. 4: Experimental apparatus

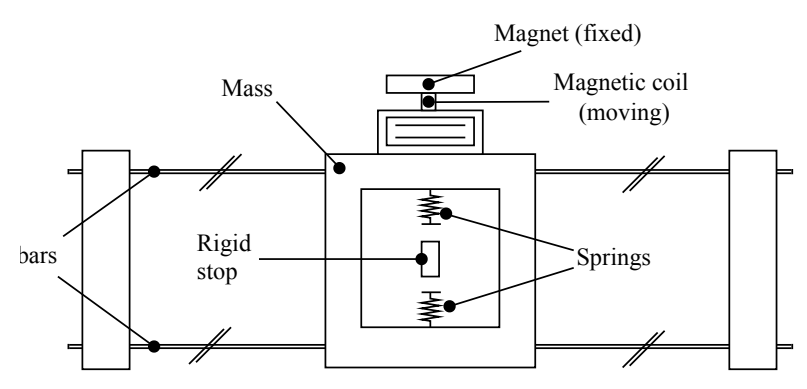

(a) Experimental configuration.

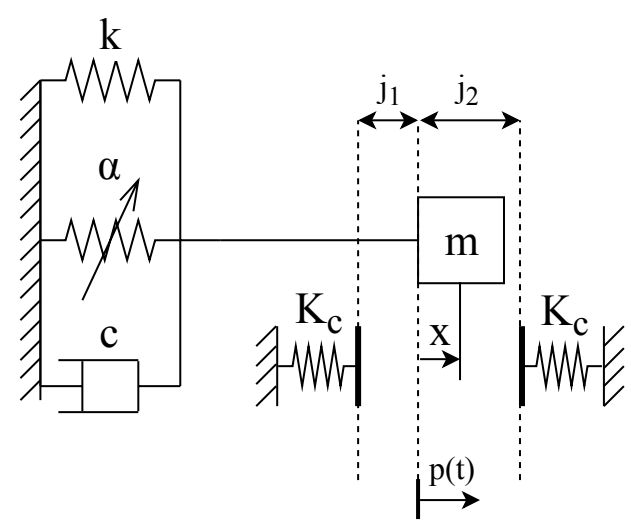

(b) SDOF model.

Fig. 5: Idealized system

and depicted in Fig. 5 b).

Nonlinear terms, included in $f_{\mathrm{NL}}$, come from two distinct effects:

1. During vibration, the clamped bars are bent perpendicularly to their length, which produces tension. The pro- jection of this force onto the direction of motion gives rise to a cubic stiffness term $f_{\mathrm{NL}, \mathrm{g}}=\alpha x^{3}$, where $\alpha$ is a constant depending on the geometry of the bars and their Young modulus.

2. A piecewise-linear stiffness induced by the clearances: $f_{\mathrm{NL}, \mathrm{c}}=K_{c}\left[\left(x+j_{1}\right) H\left(x+j_{1}\right)+\left(x-j_{2}\right) H(x-j 2)\right]$. $H(\cdot)$ represents the Heaviside step function.

Note that, in general, some amount of dissipation can be expected due to contact, which would require the inclusion of a piecewise-linear damping force in our model as was done in [35]. However, a combination of force measurements and free-oscillation tests revealed this effect to be negligible compared to modal damping, and thus it was omitted from the present model.

Before proceeding further, equation (38) is recast into non-dimensional form. Introducing the following dimensionless quantities:

$$
\begin{gathered}
x(t)=j_{1} \bar{x}(t), \omega_{0}=\sqrt{\frac{k}{m}}, \quad \tau=\omega_{0} t, c=2 \omega_{0} m \zeta, \omega=\bar{\omega} \omega_{0}, \\
p=k j_{1} \bar{p}, \quad j_{2}=j_{1} \bar{j}, \quad \alpha=\bar{\alpha} \frac{k}{j_{1}^{2}}, \quad K_{c}=\bar{K}_{c} k
\end{gathered}
$$

the equation of motion is written as:

$\bar{x}^{\prime \prime}(\tau)+2 \zeta \bar{x}^{\prime}(\tau)+\bar{x}(\tau)+\bar{\alpha} \bar{x}^{3}(\tau)+\bar{F}_{c}(\bar{x}(\tau))=\bar{p} \cos (\bar{\omega} \tau)$

where $(\cdot)^{\prime}$ represents derivation with respect to $\tau$ and the restoring force from the clearances is:

$\bar{F}_{c}(\bar{x}(\tau))=\bar{K}_{c}[(\bar{x}(\tau)+1) H(\bar{x}(\tau)+1)+(\bar{x}(\tau)-\bar{j}) H(\bar{x}(\tau)-\bar{j})]$ 
The choice of using $j_{1}$ rather than $j_{2}$ as a reference length is arbitrary and would not change the results if reversed. It is, however, convenient to introduce the ratio of clearances $\bar{j}$ as a parameter, since this provides a way to quantify the symmetry of the system. As shown next, this symmetry factor has a defining influence on the system's bifurcation behaviour.

As a final preliminary step before analysis, we replace the non-smooth contact force (41) by a regularized approximation. This is not necessary for the HBM-based continuation to succeed, as stated in Sect. 2.1. However, for stiff springs, we found that some LP were actually discontinuous bifurcations [36]. This means that, at these points, the system changes stability with one Floquet multiplier jumping over the imaginary axis instead of smoothly crossing it. Classical root-finding algorithms are then sure to fail, and a way to get rid of this inconvenience consists in introducing a regularization such as the following:

$\bar{F}_{c, r}(\bar{x}(\tau))=\bar{K}_{c}\left[\bar{x}(\tau)+\frac{1}{\pi}\left(f^{-}-f^{+}+\frac{1}{2 \sigma} f^{L}+a_{c}\right)\right]$

where:

$f^{+}=(\bar{x}(\tau)+1) \tan ^{-1}(\sigma(\bar{x}(\tau)+1))$

$f^{-}=(\bar{x}(\tau)-\bar{j}) \tan ^{-1}(\sigma(\bar{x}(\tau)-\bar{j}))$

$f^{L}=\log \left[\frac{1+(\sigma(\bar{x}(\tau)+1))^{2}}{1+(\sigma(\bar{x}(\tau)-\bar{j}))^{2}}\right]$

$a_{c}=\left[\tan ^{-1}(\sigma)-\bar{j} \tan ^{-1}(-\sigma \bar{j})-\frac{1}{2 \sigma} \log \left(\frac{1+\sigma^{2}}{1+(\sigma \bar{j})^{2}}\right)\right]$

The function $\bar{F}_{c, r}$ from (42) tends to the non-smooth contact force as $\sigma \rightarrow \infty$. In the case of the results presented hereafter, $\sigma=3 \cdot 10^{3}$ was fixed. The choice of this value was made after numerical tests, which showed that further increasing $\sigma$ beyond this point had a neglectable effect on the position of bifurcations.

\section{Analysis}

\subsection{Period doubling and route to chaos}

The values of parameters used in this study are summarized in Table 1. Numerical calculations were carried out using a MATLAB implementation of the AFT-HBM with pseudoarclength continuation as described in Sections 2 and 3. The results of HBM calculations can greatly depend on the number of harmonics used in the approximation. Thus, the choice must be made carefully. This is especially true in the case of stiff problems like the one at hand: whereas the frequency content of displacements is usually limited to a few harmonics, a higher number is generally required to appropriately represent the contact forces. For the present case, a simple convergence criteria based on the energy of neglected harmonics was implemented as proposed in [37]. On this basis, it was found through numerical tests that choosing $\mathrm{H}=15$ was enough to guarantee that higher harmonics had a neglectable effect on even the most nonlinear responses for the present system, which correspond to the strongly forced, asymmetric configuration. For the sake of simplicity, the same number of harmonics $(\mathrm{H}=15)$ was used for all tested cases (even though convergence could be obtained with a lower H), with 256 sampling points used for the AFT algorithm. It should be stressed that using such a high number of harmonics is not needed for frequency intervals away from resonances, where the response amplitudes (and thus the magnitudes of nonlinear forces) are small. However, this does not substantially increase computation times in this case since only one degree of freedom is considered in this model. Of course, more refined harmonic selection strategies should be adopted for higher-dimensional systems.

Table 1: Parameter values for Section 5.1.

\begin{tabular}{cccccc}
\hline$\zeta$ & $\bar{\alpha}$ & $\bar{\omega}$ & $\bar{p}$ & $\bar{K}_{c}$ & $\bar{j}$ \\
\hline 0.03 & 0.16 & {$[1.3 ; 4.0]$} & {$[0.0 ; 2.0]$} & {$[0.0 ; 6.0]$} & {$[0.0 ; 1.0]$} \\
\hline
\end{tabular}

Following [17], we focus our interest on the post-resonant $(\bar{\omega}>1)$ behaviour of the system as a function of the forcing frequency.

\section{Frequency-response curves}

The contact stiffness is initially set to $\bar{K}_{c}=4.7$. Figure 6 shows two frequency response curves, corresponding to perfect symmetry $(\bar{j}=1)$ and "maximum" asymmetry $(\bar{j}=0)$, for a weak forcing case: $\bar{p}=0.55$. The results are quite different: while they have comparable peak amplitudes and both show a bi-stable zone between two LP bifurcations, the locations of these points differ. More importantly, an additional unstable region appears in the asymmetric case for excitation frequencies beyond twice the natural frequency. Two period doubling bifurcations, which are absent in the symmetric case, border this region, where the system enters a sub-harmonic vibration regime. This new branch has also been computed using the branching algorithm of Sect. 3.3, and it can be observed that it contains no bifurcations at this forcing level. The same qualitative behaviour as the main resonance is displayed, with a bi-stable zone generated by a hardening effect.

Now we consider the case of a strong forcing: $\bar{p}=1.7$. As the symmetric case showed no qualitative changes with respect to the former case, it is not presented here. On the other hand, as seen in figure 7, the sub-harmonic branch of the system with asymmetric gaps contains two additional PD bifurca- 
tions. Between them, the 2T-periodic solutions are unstable, giving rise to 4T-periodic motions.

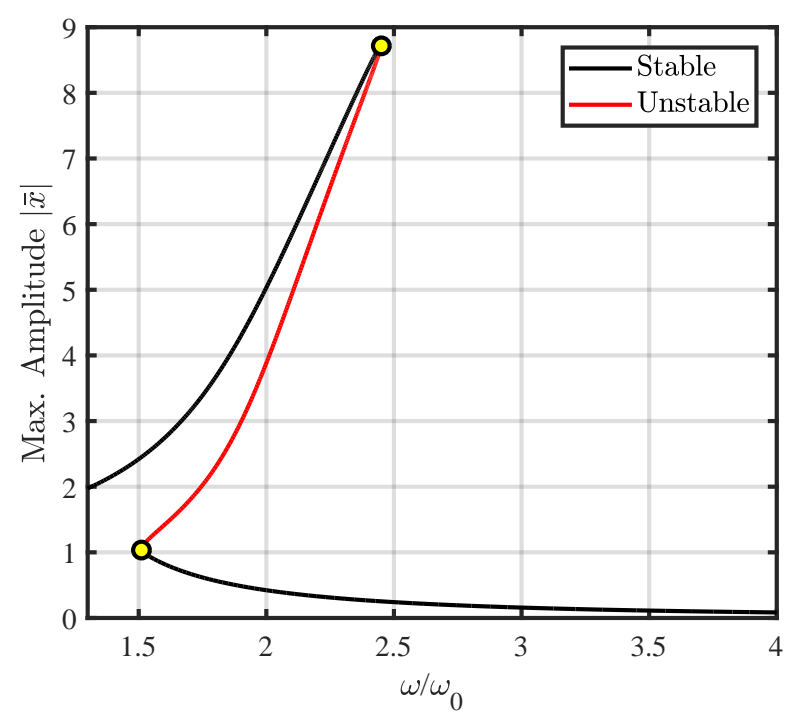

(a) Symmetric case $(\bar{j}=1)$

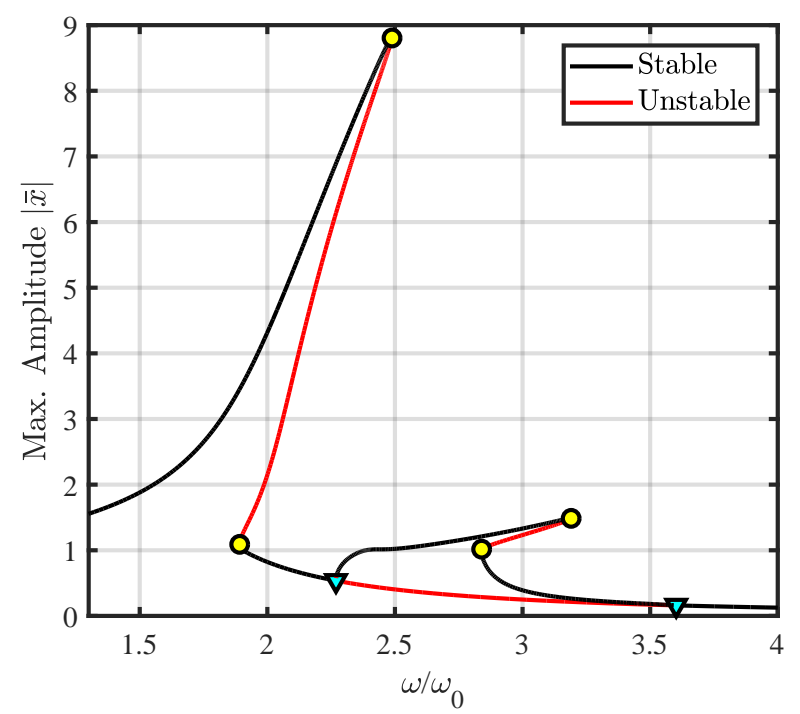

(b) Asymmetric case $(\bar{j}=0)$

Fig. 6: Frequency-response curves, weak forcing. (॰: Limit Point; $\nabla$ : Period-Doubling bifurcation point)

The cycles corresponding to the points labelled $\mathbf{A}$ and $\mathbf{B}$ in Fig. 7 (respectively before and after the lowest-frequency PD bifurcation) are shown in phase space in Fig. 8. The latter can be seen to contain an additional loop when compared to the former. Thus, in the span of one forcing period, the whole of cycle $\mathbf{A}$ is described, whereas only half of $\mathbf{B}$ is. This provides visual evidence of a double-period response.

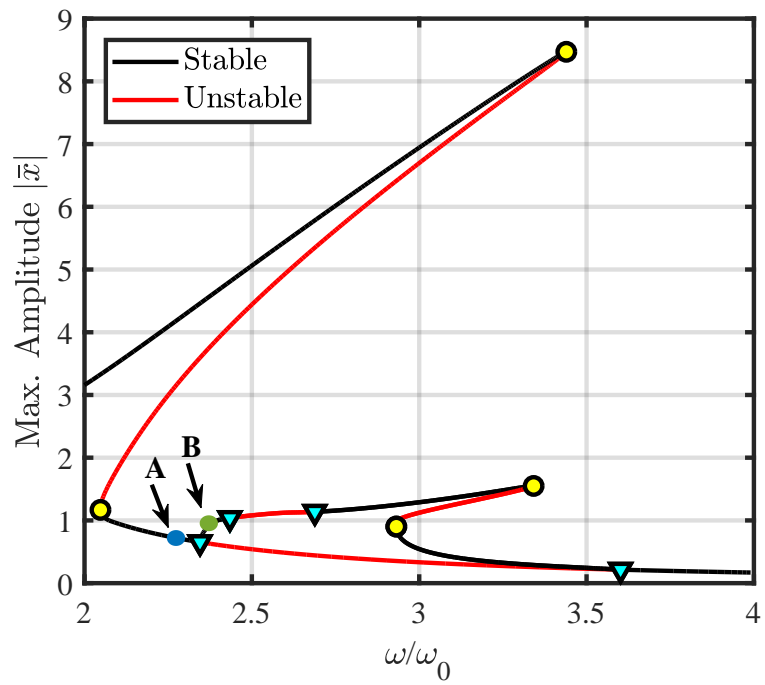

Fig. 7: Successive PD bifurcations in the asymmetric configuration, strong forcing.

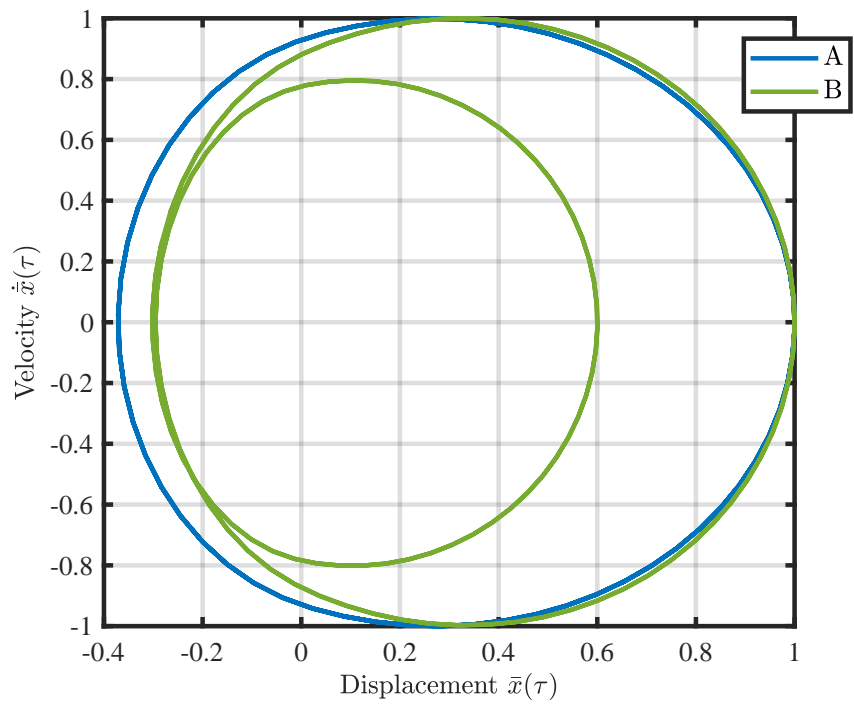

Fig. 8: Phase space plots of cycles $\mathbf{A}$ and $\mathbf{B}$ from Fig. 7.

\section{Forcing amplitude}

The PD bifurcations presented above for $(\bar{j}=0)$ were tracked with respect to the forcing amplitude $\bar{p}$ by means of the continuation algorithm presented in Sect. 3.4. The resulting curves, as well as their projection on the codimension-2 plane $(\bar{\omega}, \bar{p})$, are presented in Fig. 9. For visualization purposes, response curves at different values of $\bar{p}$ are included as well.

It can be seen that the first pair of PD points happens independently of the forcing amplitude over the considered range, thus implying that this phenomenon is not driven by external forcing. On the contrary, the stability boundary corresponding to the second stage of the cascade, labelled 


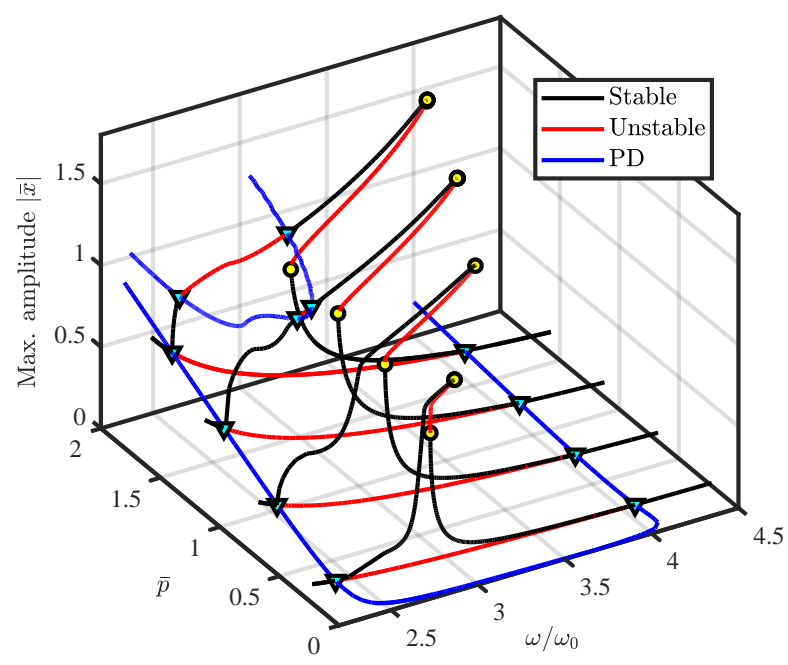

(a) PD bifurcation tracking

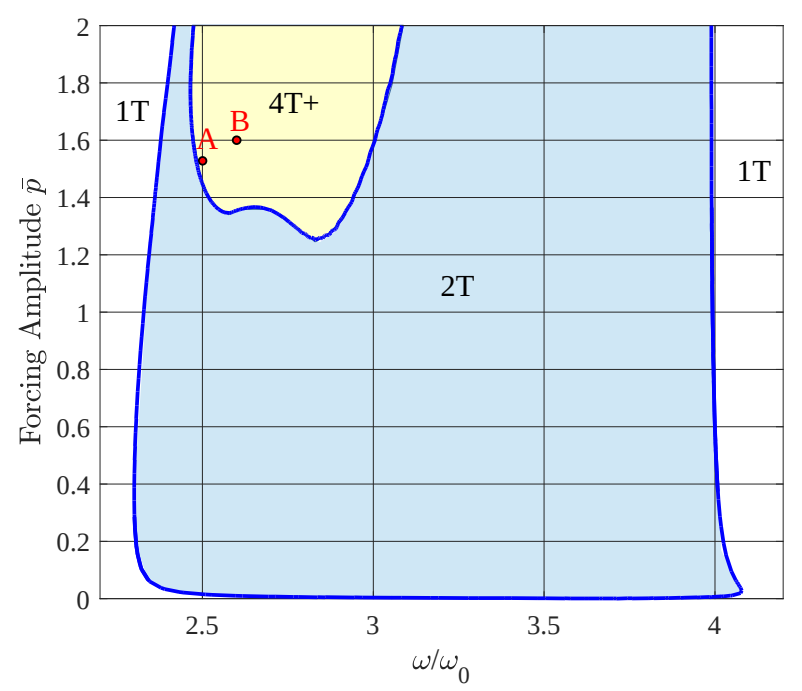

(b) Period doubling boundaries on $(\bar{\omega}, \bar{p})$ plane. Labels indicate the periodicity of solutions in each zone.

Fig. 9: Forcing amplitude as tracking parameter.

"4T+" (solutions whose period is at least 4T), only exists for high forcing amplitudes.

\section{Contact stiffness}

While varying the contact stiffness in a continuous fashion is evidently infeasible in practice, this can be done with ease by continuation methods. The four PD bifurcations from Fig. 7 were tracked with respect to the contact stiffness $\bar{K}_{c}$, as shown in Fig. 10, with forcing amplitude $\bar{p}=1.7$.

From these curves, it is easily seen that the first period doubling is quite sensitive to contact stiffness, in contrast with the case of forcing amplitude, since the location of bifurca-

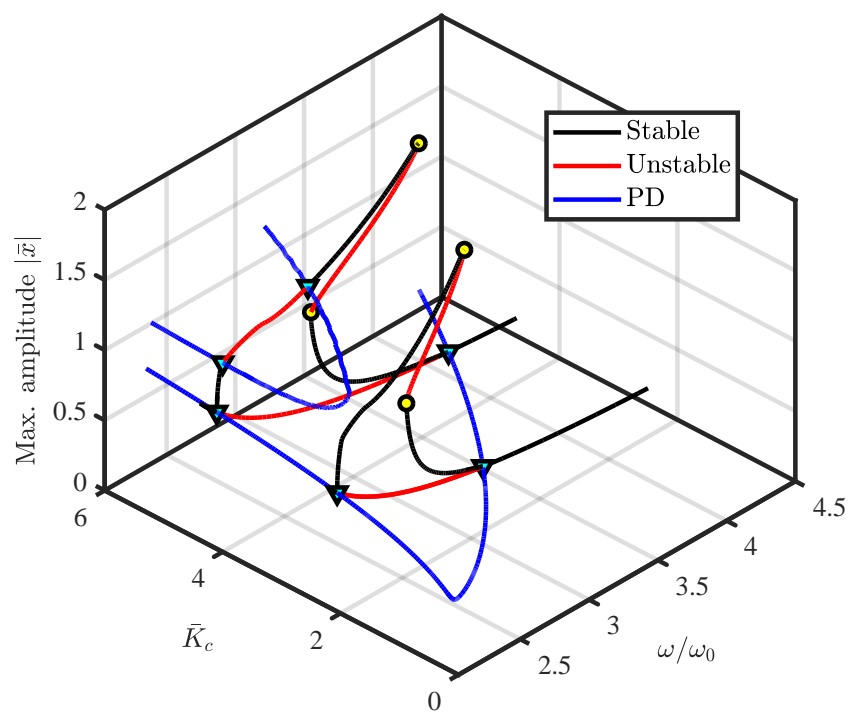

(a) PD bifurcation tracking

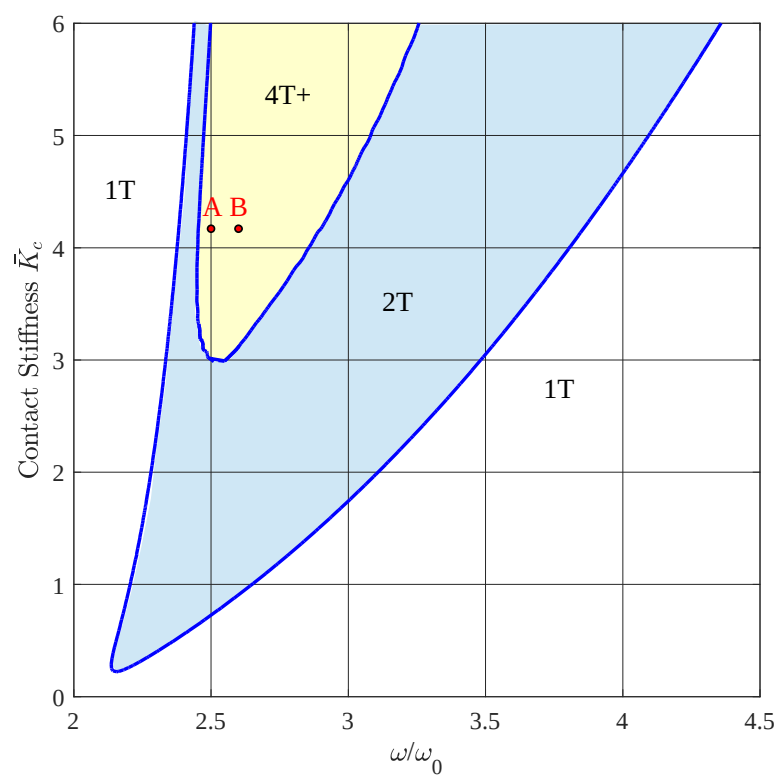

(b) Period doubling boundaries on $\left(\bar{\omega}, \bar{K}_{c}\right)$ plane. Labels indicate the periodicity of solutions in each zone.

Fig. 10: Contact stiffness as tracking parameter.

tion points changes significantly as this parameter is varied. Nonetheless, consider the local extremum close to null contact stiffness. From physical grounds, a value of zero simply indicates an absence of springs, and the system reduces to a typical, symmetrical Duffing oscillator in such a case. It is seen here that the boundary folds back shortly before reaching the $\omega$-axis, but we can state that period doubling occurs over practically the whole interval of contact stiffness values, and so this parameter is also not the main trigger for bifurcation in this case. 


\section{Chaos}

So far, we have confirmed the well-understood fact that high inputs of energy by external forcing, as well as increasingly stiff contacts, have a tendency to promote bifurcation. Successive sub-harmonic branching such as the one observed here hints to the presence of a sequence of PD bifurcations leading to chaos. It is, of course, not possible to use harmonic balance to compute chaotic regimes, since these are aperiodic by definition. Nonetheless, the search for parameter regions associated with chaos can be limited to those in the neighborhood of high-period boundaries. An example of this can be seen in Fig. 11 . Here, the values $\left(\bar{p}, \bar{K}_{c}\right)=(1.6,4.2)$ were fixed and a constant-acceleration Newmark scheme was used to numerically integrate the equation of motion over a range of frequency excitation values. A Poincaré map was then established by sampling the response signal (amplitudes and velocities) at intervals equal to the excitation period. Integration was carried out over 1000 periods to ensure that the steady state had been reached, and the amplitudes of the last 100 samples are plotted as a function of frequency.

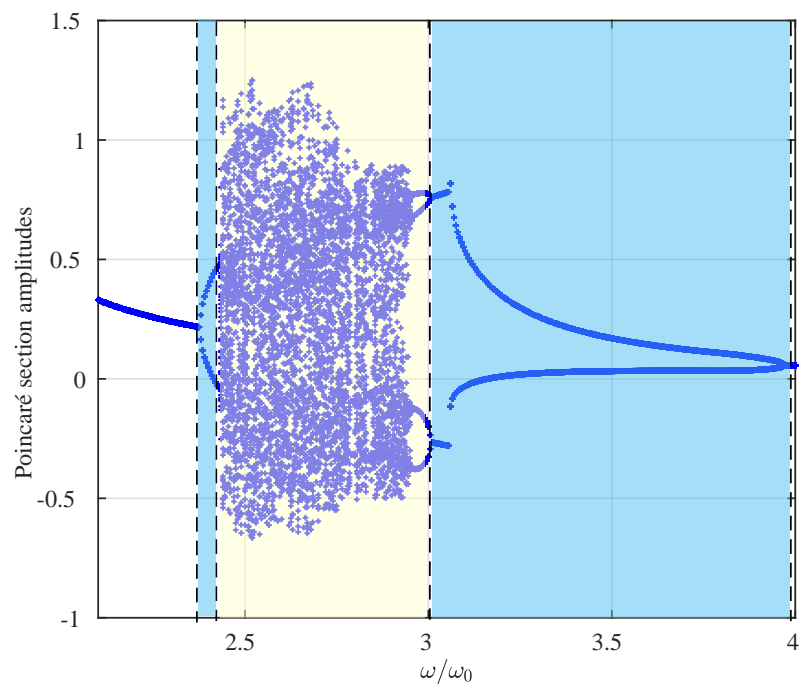

Fig. 11: Bifurcation diagram. Blue and yellow zones correspond to predicted $2 \mathrm{~T}$ and 4T-periodic regimes, respectively.

The results observed in this figure match the predictions from Figs. 9 and 10 in terms of bifurcation behaviour. Moreover, regions of erratic responses can be found within the predicted $4 \mathrm{~T}+$ frequency interval. It can be noted that, while 4T-periodic responses are clearly visible, the transition to chaos is rather abrupt, and thus additional steps in the perioddoubling cascade $(8 \mathrm{~T}, 16 \mathrm{~T} . .$.$) can only be observed over$ extremely narrow ranges of forcing frequency.

Fig. 12 shows the region of predicted and experimentally observed chaos for the same system from de Langre and Lebreton's paper [17], with respect to forcing amplitude. It can be seen that the main boundary is both quantitatively and qualitatively similar to the 4T+ boundary of Fig. 9 (b), which once again suggests a rapid transition once the period doubling sequence is triggered. Furthermore, this highlights the usefulness of bifurcation tracking as a predictive tool, as it yields results close to those obtained with the more computationally intensive method employed in the cited paper.

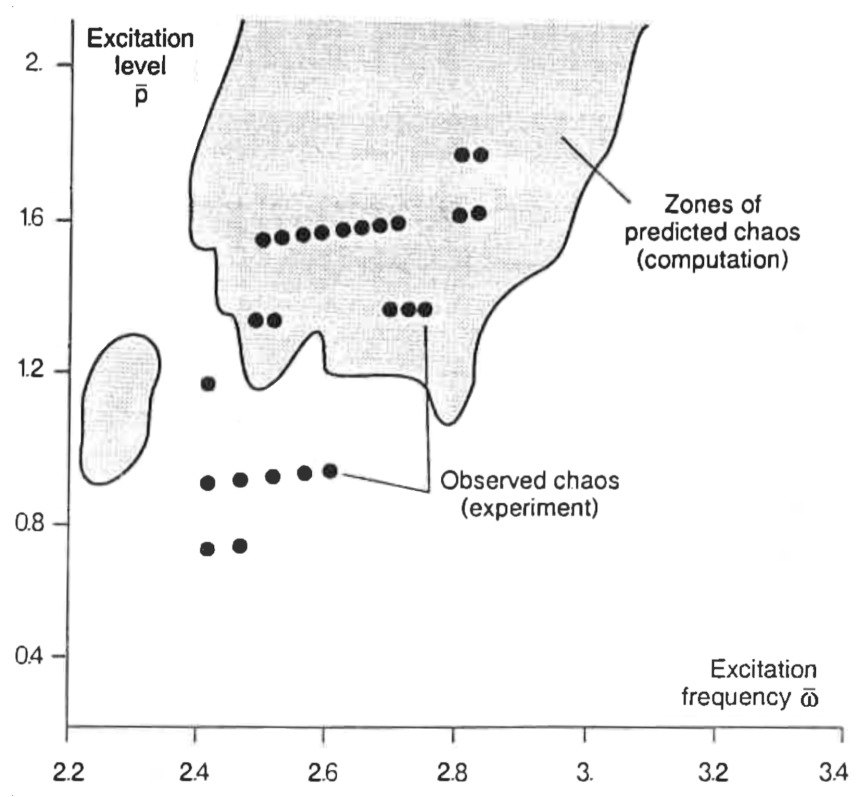

Fig. 12: Region of predicted chaos, from [17].

The point labelled $\mathbf{A}$ in Figs. 9 and 10, for which a fractal attractor was reported in [17], is included in this zone as well. Other points inside this boundary also exhibit aperiodic motion, an example of which is seen in Fig. 13. These results were obtained by fixing parameter values to those of point $\mathbf{B}$ : $\left(\bar{\omega}, \bar{p}, \bar{K}_{c}\right)=(2.6,1.6,4.2)$.

The pattern formed on the Poincaré section of Fig. 13 (b) strongly suggests a chaotic nature, as the orbit is confined to a specific region in phase space (implying the presence of an attractor) and no periodicity is evident. To verify that this response is indeed chaotic, its leading Lyapunov exponent, $\lambda_{L}$, was computed. As explained e.g. in [38], a positive value of this quantity indicates that two initially close trajectories diverge exponentially fast, which is a signature of chaos. Thus, letting $\delta(\tau)$ represent the instantaneous distance between these in phase space, we may write:

$\delta(\tau) \simeq \delta(0) e^{\lambda_{L} \tau}$ 


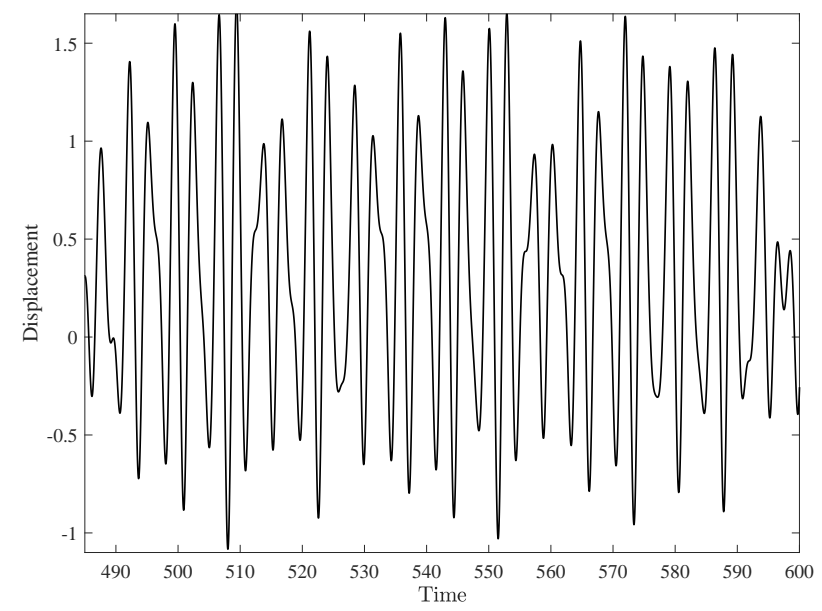

(a) Time series over 50 excitation periods.

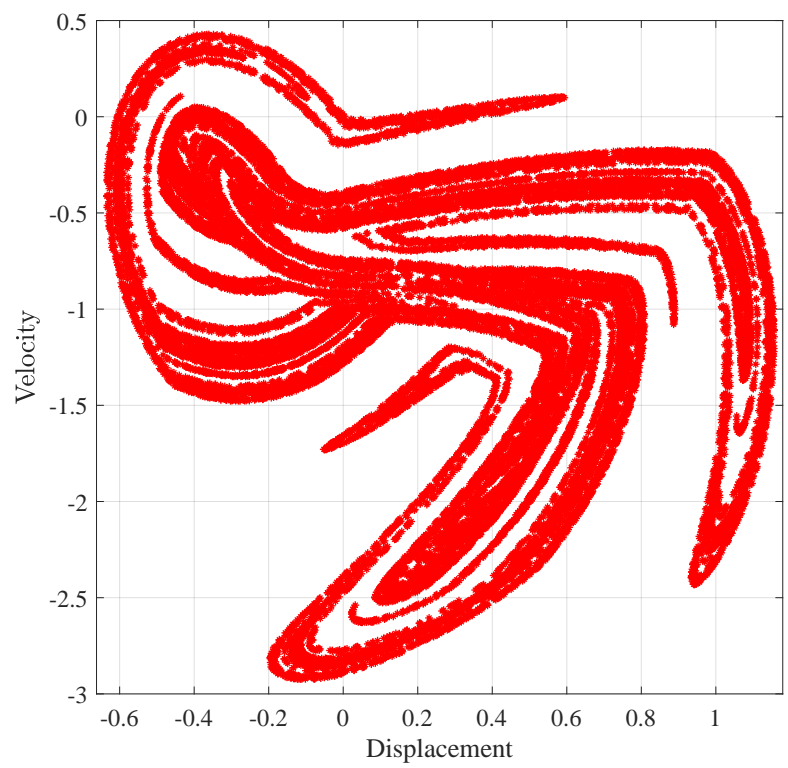

(b) Poincaré section with 5000 samples.

Fig. 13: Response at point $\mathbf{B}$ in Figs. 9 and 10.

Fig. 14 shows the time history of $\delta(\tau)$ as obtained from two response signals: the first one, corresponding to Fig. 13, was computed for null initial conditions (position and velocity equal to 0 ), whereas a perturbation of magnitude $10^{-14}$ was added to initial position for the second one. Sensitivity to initial conditions is clearly evidenced by the exponential growth of the distance with time at a rate approximately equal to $\lambda_{L}=0.1918$. On the other hand, the distance can be seen to saturate, since the trajectories pertain to an attractor of fixed length and thus cannot separate indefinitely.

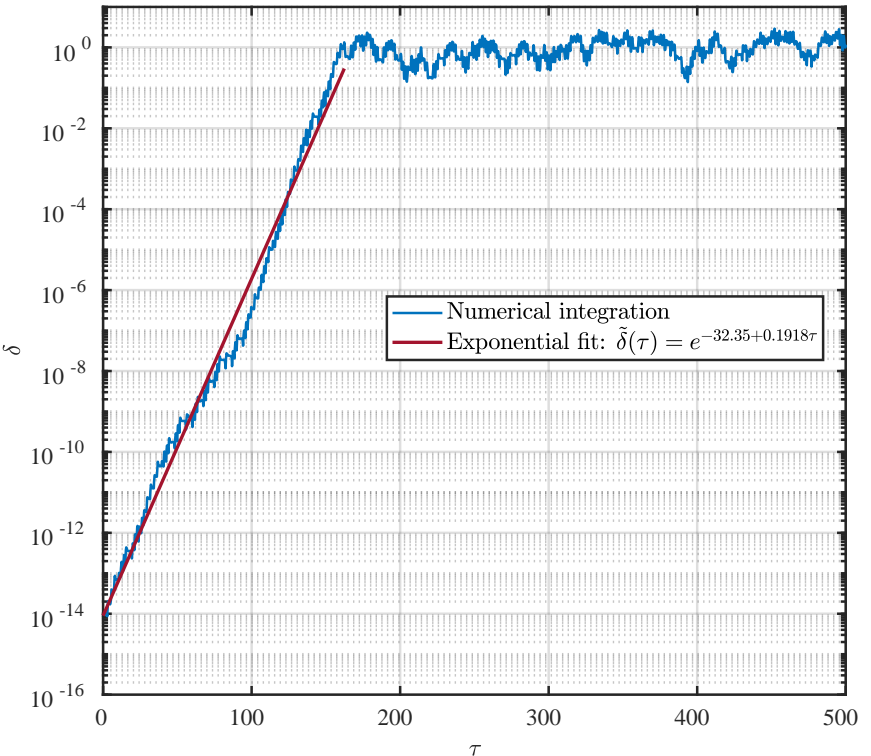

Fig. 14: Distance between two signals with close initial conditions.

\subsection{Part 2: Symmetry and sub-harmonic isolas}

Previous studies of this system focused on the two extreme cases $\bar{j}=1$ and $\bar{j}=0$. There are two reasons for this: firstly, from an experimental perspective, they are the simplest configurations to implement. Fine-tuning the value of $\bar{j}$ in practice is quite a delicate task, and extreme values are thus more convenient. Secondly, from a phenomenological point of view, it is well-known fact that asymmetry has the tendency to promote bifurcation. Hence, if the objective is solely to observe chaotic motions, it is the "most asymmetric" configuration which offers the optimal conditions. Yet, as observed in the previous section, even the first period doubling of the cascade is absent in the symmetric case, and so no chaos at post-harmonic excitation frequencies may be observed by this route. Therefore, asymmetry is a necessary condition for period doubling. It should be noted that, generally speaking, initially symmetric systems can have their symmetry broken through a regular BP and then undergo period doubling along the asymmetric branches. However, for the parameter intervals considered in this study, this behaviour was not observed. An interesting question which immediately arises is then: how asymmetric must the clearances be in order for period doubling to be possible?

Table 2: Parameter values for Section 5.2.

\begin{tabular}{cccc}
\hline$\zeta$ & $\bar{\alpha}$ & $\bar{p}$ & $\bar{K}_{c}$ \\
\hline 0.15 & 0.03 & 0.55 & 2.48 \\
\hline
\end{tabular}


For the remainder of this section, we set the parameters at the values shown in Table 2, which correspond to the characteristics of the current experimental setup. Figure 15 shows PD bifurcation tracking with respect to the symmetry factor, starting from $\bar{j}=0$, while Fig. 16 shows a projection of this curve on the parameter plane $(\bar{\omega}, \bar{j})$.

It is clear that $\bar{j}$ has a very strong influence on period doubling, since the first stability boundary is only defined over a limited range of values: the bifurcation curve has a single local extremum $(\bar{\omega}, \bar{j})=\left(\bar{\omega}_{\text {det }}, \bar{j}_{\text {det }}\right)$, whose concavity is opposite to those observed while tracking PD bifurcations with respect to other parameters. At this point, two PD bifurcations coalesce, but the associated sub-harmonic resonance peak does not disappear: rather, it forms a closed loop which is tangent to the main branch. A further increase in $\bar{j}$, towards symmetry, causes the loop to detach and form an isola. Of course, one can also see the situation in the reverse way: as the system strays further away from symmetry, an isola approaches the main branch until merging at a codim-2 local extremum of the PD stability boundary, localized by means of the extended system presented in Sect. 3.5.

Thinking back to the theory of Sect. 3.6, this point should be a LP when calculated on the augmented Fourier basis $\mathbb{T}_{s}^{H}(\omega t)$. It can therefore be used, after changing bases, as a starting point for the LP tracking algorithm with respect to $\bar{j}$ (step 4 of the algorithm of Sect. 3.6). This LP tracking shows that, for any $\bar{j}$ larger than the critical value $\bar{j}_{\text {det }}$, the subharmonic branch detaches and continues to exist as an isola over a certain range of $\bar{j}$. The existance of LPs on the isolas allows the use of bifurcation tracking, which in turn reveals the presence a codim-2 local extremum at $\bar{j}=\bar{j}_{\text {max }}$ where the two branches coalesce and the isola collapses into a point corresponding to a sub-harmonic isola centre. At this state, the system is "critically symmetric", so that the contribution of the asymmetric harmonics from the nonlinear forces is just important enough to induce bifurcation. For $\bar{j}>\bar{j}_{\max }$, the isola does no longer exist. From these observations, it results that bifurcation tracking (LPs and PDs) is an efficient tool which provides, with few computations, the curves delimiting the regions of existence of 2-T periodic solutions as well as 2T-isolas. This information is very useful for choosing the parameters of the experimental setup as shown in following sections, and is of great importance in the larger context of mechanical system design.

\subsection{Global dynamics}

Isolated sub-harmonic resonances have been shown to exist for system (40) for certain parameter combinations and over determined ranges of excitation frequency. Moreover, since they contain a stable branch, they can theoretically be reached by choosing appropriate initial conditions or by adding a perturbation to a stable solution on the main branch. However,

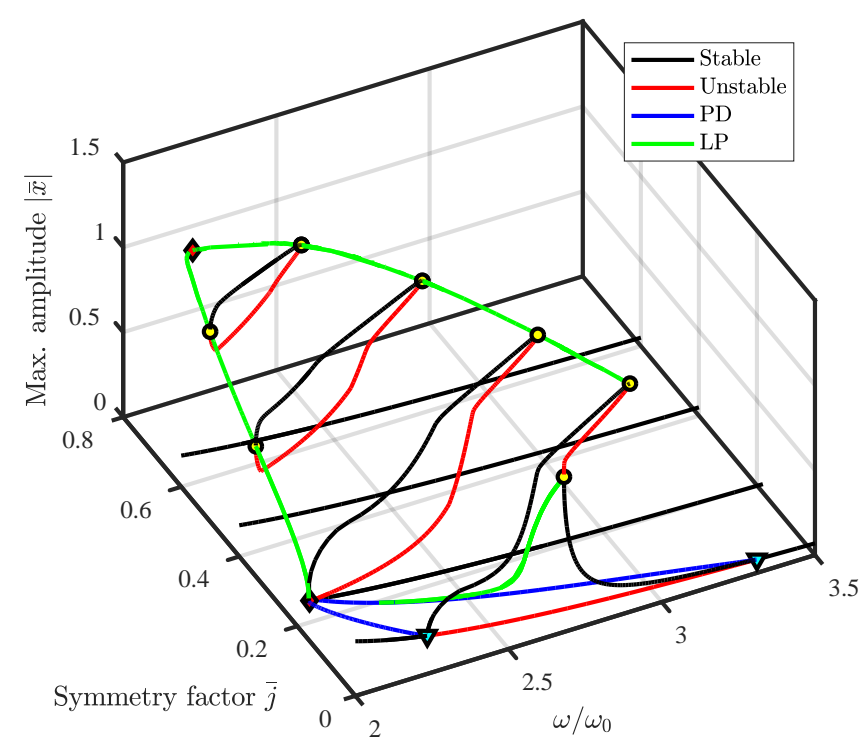

Fig. 15: Symmetry factor as tracking parameter.

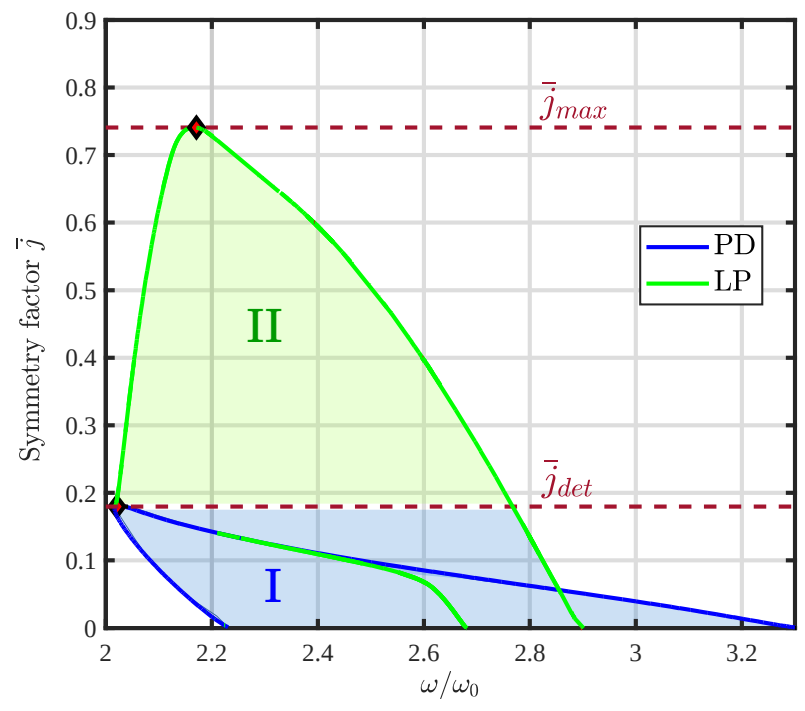

Fig. 16: Tracking of limit points from an isola merging point. Region I (blue): 2T-periodic solutions.

Region II (green): 2T-isolas.

in practice, the basin of attraction associated to the isola can be small compared to that of the T-periodic solution, in which case it would be difficult to observe them experimentally. To assess the robustness of the isolas, we have numerically computed basins of attraction for fixed $(\bar{\omega}, \bar{j})$ pairs within their predicted range of existence, i.e. for $\bar{j} \in\left[\bar{j}_{\mathrm{det}}, \bar{j}_{\text {max }}\right]$ as shown in Fig. 16. For (non-dimensional) initial conditions $\left[x_{0}, \dot{x}_{0}\right] \in[-2,2] \times[-2,2]$, the equation of motion (40) was 
integrated over several excitation periods until a steady state was established. Using the absolute norm:

$x_{\max }=\|\bar{x}(\tau)\|=\max _{\tau=0, \ldots, 2 \pi}\{|\bar{x}(\tau)|\}$

contour plots $x_{\max }=f\left(x_{0}, \dot{x}_{0}\right)$ were constructed. Fig. 17 shows the results obtained for $(\bar{\omega}, \bar{j})=(2.09,0.5)$.

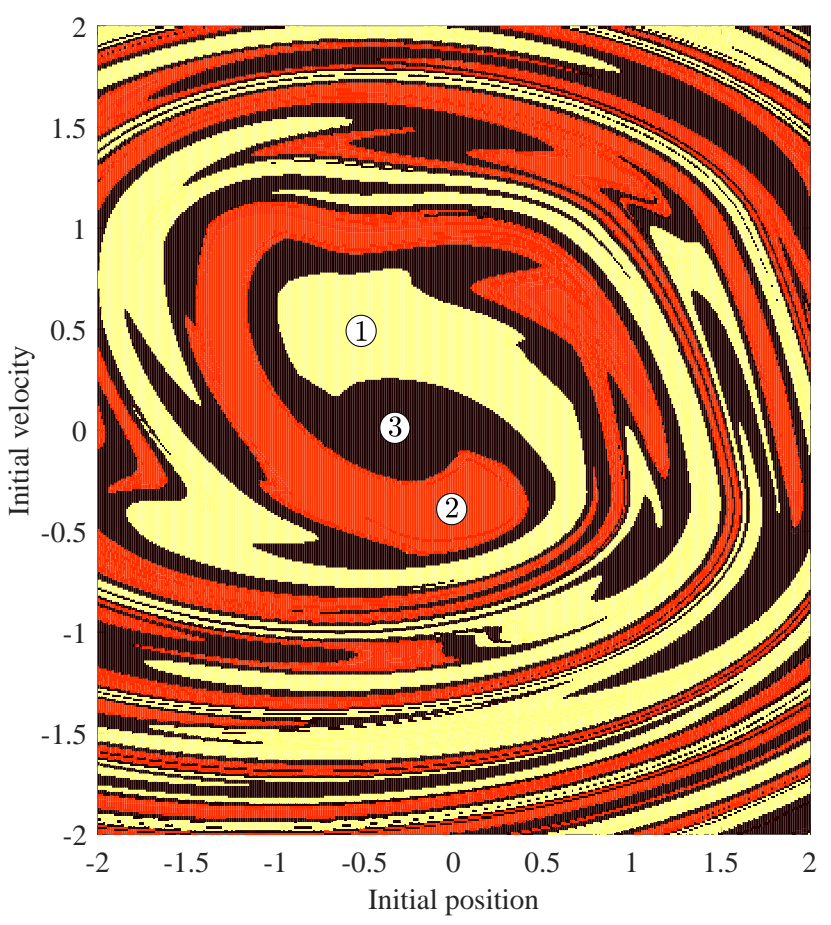

Fig. 17: Basins of attraction, showing coexistence of three regimes for $\omega / \omega_{0}=2.09$

Three basins are clearly visible, two of which correspond to the low and high-amplitude regimes in the bi-stable zone of the main resonance as in a typical Duffing-like oscillator. The remaining basin is associated with the sub-harmonic regime on the isola, as illustrated by Fig. 18. No attractor seems to be particularly dominant, i.e., for random initial conditions, the chances of finding any one of this regimes are roughly equally likely, which means that these isolas must be robust, thus observable. For completeness, Fig. 19 shows the harmonic content of the three numerically calculated solutions. As expected, the low-amplitude (non-resonant and non-impacting) regime is mono-harmonic, the isolated one is predominantly sub-harmonic and the large-amplitude, nonlinear resonant response shows slight harmonic distortion as well as a static component due to asymmetry.

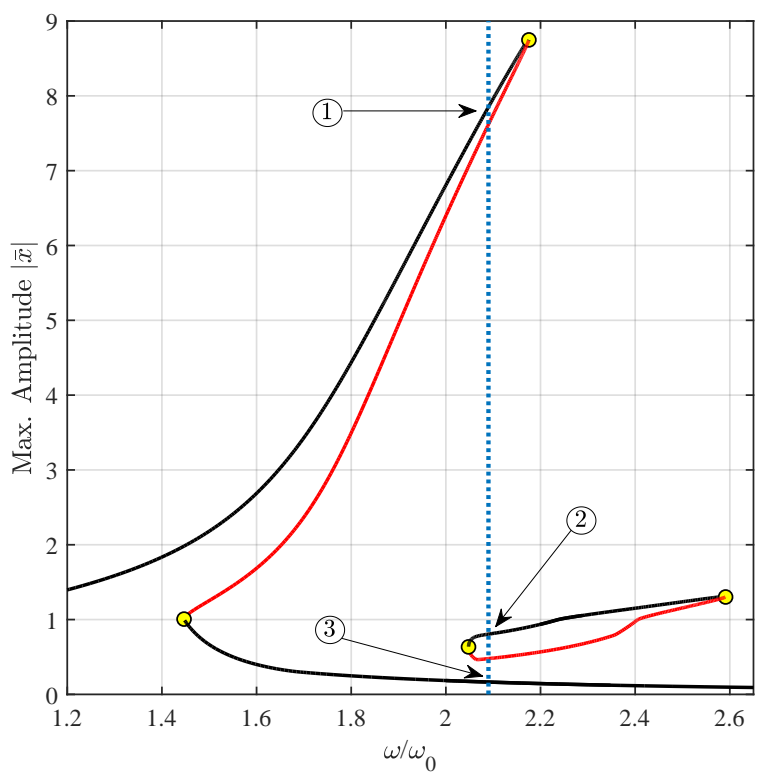

Fig. 18: Frequency-response curve for $\bar{j}=0.5$

\subsection{Experimental sub-harmonic isolas}

Knowing, from last section, that isolated sub-harmonic regimes indeed exist for this system, they were sought experimentally. The transverse displacement of the central mass is directly measured by a LDA-05 laser vibrometer, whereas velocity data is obtained a posteriori by finite differences.

Foremost, Fig. 20 shows the experimental frequency response function in a neighbourhood of $\omega / \omega_{0}=2$, obtained via the Virtual Step-Sine (VSS) method. Briefly stated, a sine wave is stepped through the frequency range of interest and the ratio of input and output signals is displayed as the response magnitude. It should be noted that this calculation is actually equal to the ratio of the cross-spectrum and the reference auto-spectrum, which means that only the fundamental harmonic of the response is kept by this procedure. Hence, after conversion to units of length and non-dimensionalization, the quantity obtained and plotted in the vertical axis is denoted $\left|X_{1}\right|$, and can be directly compared to the predicted non-dimensional amplitudes of Fig. 18.

Two ${ }^{5}$ sets of data are plotted in Fig. 20. The solid line corresponds to a usual stepping with increasing forcing frequency, and no bifurcation is present. Then, starting from a steady oscillating state within the predicted interval of isola existence, the system was perturbed by a gentle tap, and a transition to a different regime, with higher oscillation amplitude and isolated from the main response curve, was observed. Sine-stepping from this point yielded the dashed

5 The large-amplitude resonance peak seen in Fig. 18 could be observed as well by applying a much stronger perturbation, but it was omitted from the figure as it is of no interest to the phenomenon of sub-harmonic isolas. 


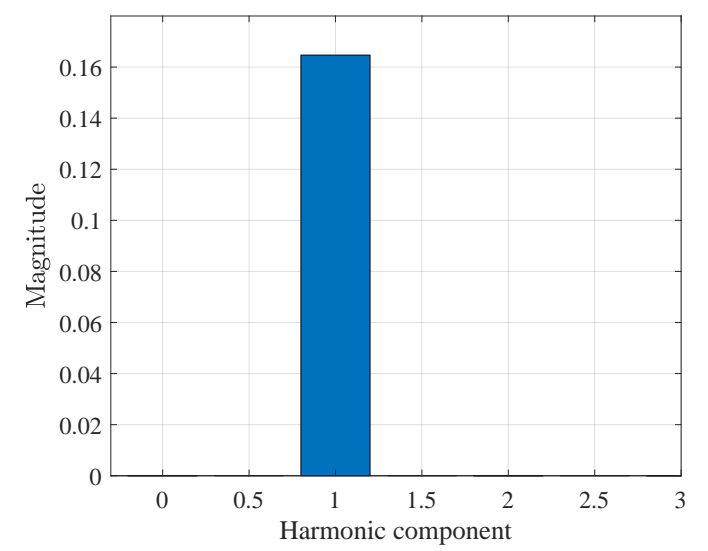

(a) Low amplitude, T-periodic regime.

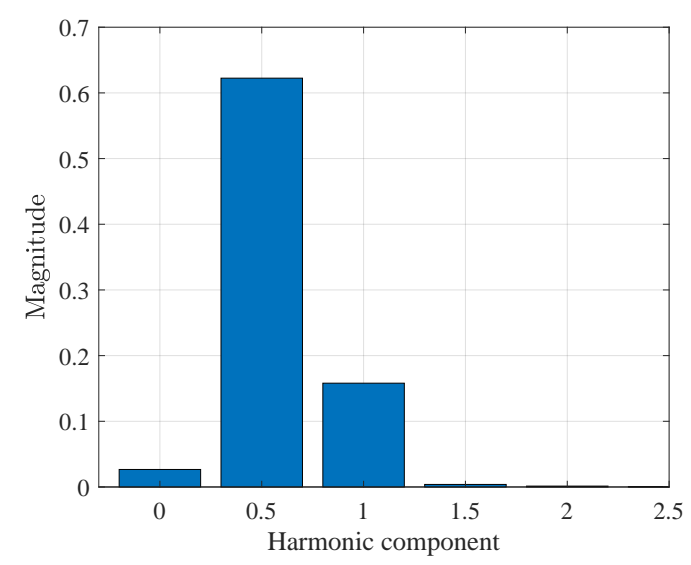

(b) Isolated 2T-periodic regime.

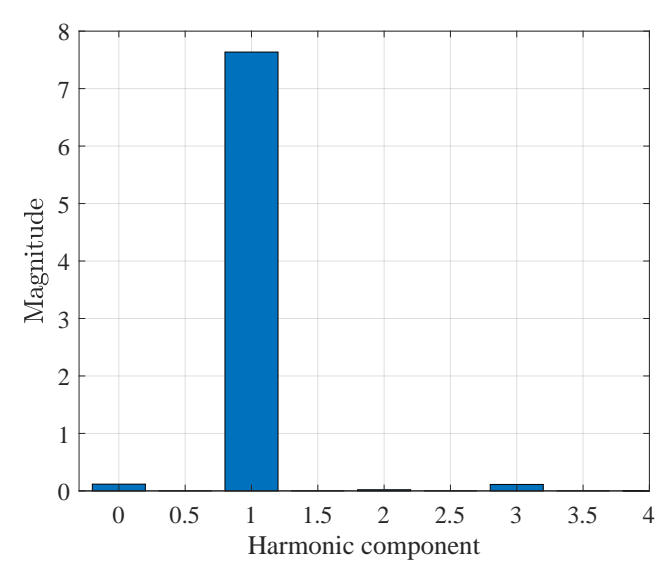

(c) Large amplitude, T-periodic regime.

Fig. 19: Frequency content for $\omega / \omega_{0}=2.09$

line. Care must be taken in the interpretation of this figure: even though it may resemble figures such as 6 , where period doubling happens on the main response branch, this is not the case. Indeed, the main branch loses stability between two PD bifurcations, and so the experimental stepping would only capture one continuous, stable branch whose frequency content changes qualitatively over an interval. In contrast, here two distinct stable branches were captured.

Within the isola, the magnitude of $X_{1}$ drops. However, as shown in Fig 19 (b), the dominant frequency component should be not the fundamental harmonic but the $\frac{1}{2}$-th one on the predicted isola. Likewise, the response on the isola should have a greater amplitude than the harmonic one on the main response curve.

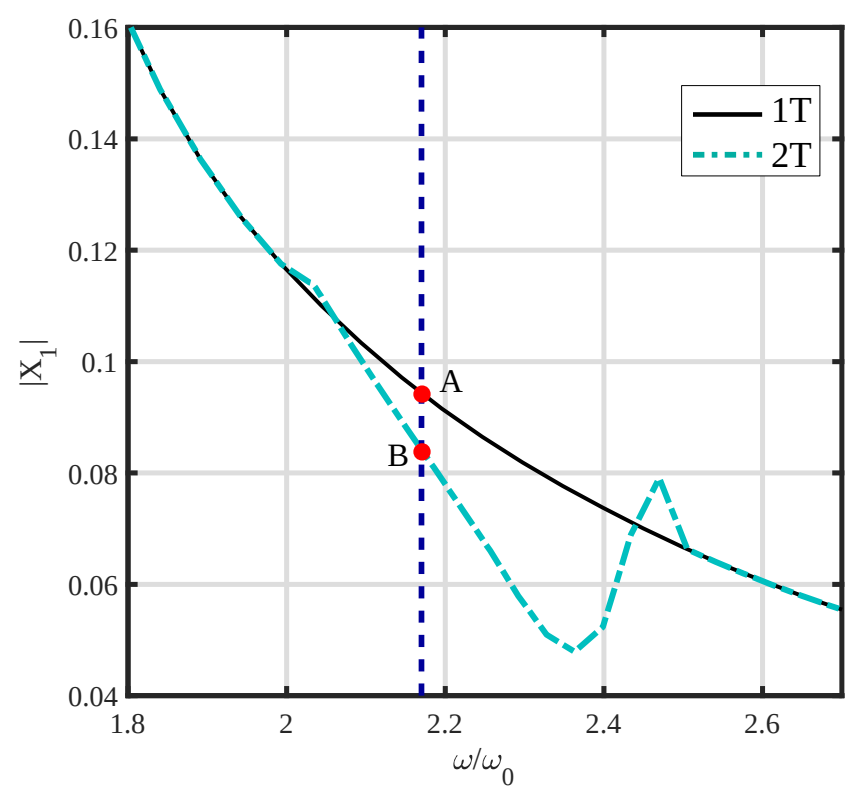

Fig. 20: Fundamental harmonic of the experimental frequency response. Coexisting regimes for $\omega \in[2.03,2.51]$.

For a fixed frequency of $\omega / \omega_{0}=2.17$, represented by the vertical line in Fig. 20, Fig. 21 shows time series of the two output regimes, over two excitation periods ${ }^{6}$. Clearly, the response on the isola it at half the excitation frequency, and in agreement with the predicted quantitative and qualitative computations from Figs. 18 and 19.

\section{Concluding remarks}

In this work, an original extended system for calculating period-doubling bifurcations was proposed. This includes their detection, localisation and tracking, as well as a simple algorithm for switching to sub-harmonic branches. Moreover, a characterisation of local extrema of bifurcation curves was proposed in a general form and then specialized to the case of period doubling, where it is an indicator of sub-harmonic isola formation. Based on these concepts, a bifurcation study

\footnotetext{
6 In terms of non-dimensional quantities, the duration of two excitation periods is $4 \pi$.
} 


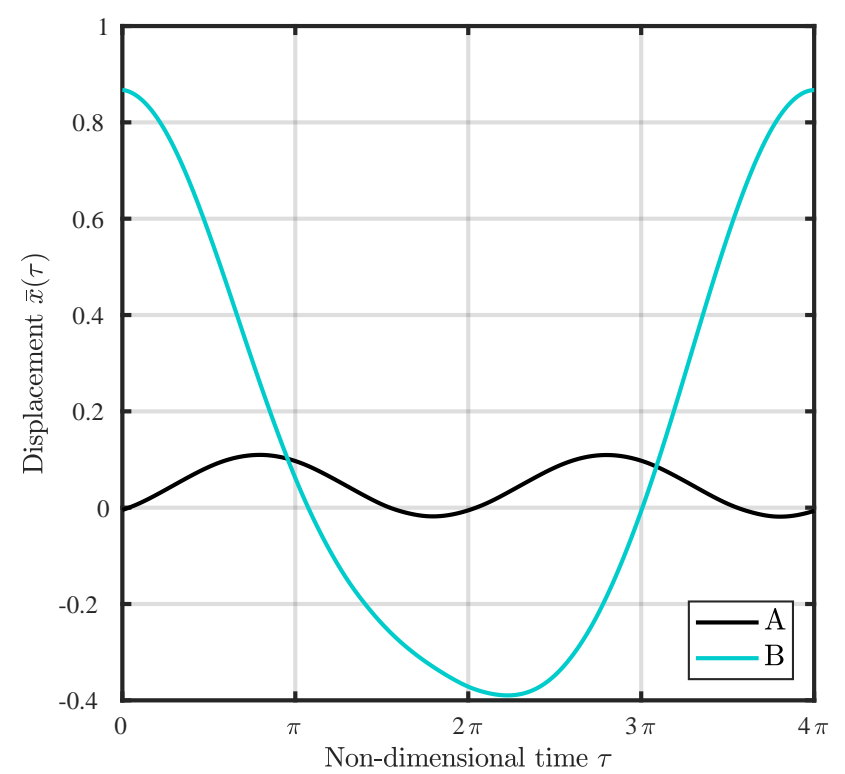

Fig. 21: Experimental time series, $\omega / \omega_{0}=2.17$. Labels correspond to points in Fig. 20.

was carried out on a forced nonlinear oscillator with clearances. While fairly simple, it is rich in bifurcation phenomena and thus offers a satisfying test case for our algorithms. Besides verifying the success of the latter in reproducing and explaining the results of previous studies, the birth of sub-harmonic isolas for the test system at a codimension-2 extremum point was explained and quantified, which is an originality of this paper. Asymmetry was identified as the main factor responsible for isola birth and merging with the main response curve, at which point period-doubling bifurcations appear. Finally, experimental results were presented which showed agreement with numerical predictions.

\section{Appendix A The Algebraic Branching Equation}

At a singular point $\left(\mathbf{X}^{0}, \omega^{0}\right)$, the Jacobian has a simple rank deficiency, which is equivalent to the existence of a single right eigenvector $\phi$ such that:

$\mathbf{R}_{\mathbf{X}}^{0} \phi=\mathbf{0}$

and a single left eigenvector $\psi$ verifying:

$\psi^{*} \mathbf{R}_{\mathbf{X}}^{0}=\mathbf{0}^{*}$

If, in addition, $\mathbf{R}_{\omega}^{0}$ belongs to the image of $\mathbf{R}_{\mathbf{X}}{ }^{0}$, the tangent is not uniquely defined at this point: this is the condition for a BP to occur. In this case, we also have:

$\psi^{*} \mathbf{R}_{\omega}^{0}=0$
Note that this implies the existence of a unique vector $\mathbf{v}$ such that:

$\left[\begin{array}{cc}\mathbf{R}_{\mathbf{X}}{ }^{0} & \mathbf{R}_{\omega}^{0} \\ \psi * & 0\end{array}\right]\left(\begin{array}{l}\mathbf{v} \\ 1\end{array}\right)=\left(\begin{array}{l}\mathbf{0} \\ 0\end{array}\right)$

Now, at any point of the frequency-response curve, the following equation holds according to (4):

$\mathbf{R}_{\mathbf{X}} \Delta \mathbf{X}+\mathbf{R}_{\omega} \Delta \omega=0$

Moreover, at a bifurcation point, this equation admits two solutions $(\Delta \mathbf{X}, \Delta \omega)_{1,2}$ corresponding to the intersecting tangent vectors. One of these, say $\left(\Delta \mathbf{X}_{1}, \Delta \omega_{1}\right)$, is already known from the previous continuation step, so:

$\mathbf{R}_{\mathbf{X}}^{0} \Delta \mathbf{X}_{1}=-\mathbf{R}_{\omega}^{0} \Delta \omega_{1}$

Taking into account (45) and (50), it is straightforward to show that the second tangent vector can be written as:

$\left(\Delta \mathbf{X}_{2}, \Delta \omega_{2}\right)=\left(\phi+\beta \Delta \mathbf{X}_{1}, \beta\right)$

where $\beta \in \mathbb{R}$ is a constant to be determined as follows. Since we are using arclength $s$ as our continuation parameter, $\mathbf{X}=$ $\mathbf{X}(s)$ and $\omega=\omega(s)$. Hence $\Delta \mathbf{X}=\frac{d \mathbf{X}}{d s}$ and $\Delta \omega=\frac{d \omega}{d s}$. We differentiate (49) with respect to arclength, pre-multiply it by $\psi^{*}$ and simplify to obtain:

$\psi^{*}\left(\left(\mathbf{R}_{\mathbf{X}} \Delta \mathbf{X}\right)_{\mathbf{X}} \Delta \mathbf{X}+\mathbf{R}_{\mathbf{X} \omega} \Delta \mathbf{X} \Delta \omega+\mathbf{R}_{\omega \omega} \Delta \omega \Delta \omega\right)=0$

Evaluating derivatives at the bifurcation point and substituting the components of the new tangent $\left(\Delta \mathbf{X}_{2}, \Delta \omega_{2}\right)$ into this expression, we get the following second-order algebraic equation for $\beta$ :

$a \beta^{2}+b \beta+c=0$

$a=\psi^{*}\left(\left(\mathbf{R}_{\mathbf{X}}^{0} \Delta \mathbf{X}_{1}\right) \mathbf{X} \Delta \mathbf{X}_{1}+2 \mathbf{R}_{\mathbf{X} \omega}^{0} \Delta \mathbf{X}_{1}+\mathbf{R}_{\omega \omega}^{0}\right)$

$b=\psi^{*}\left(\left(\mathbf{R}_{\mathbf{X}}^{0} \phi\right)_{\mathbf{X}} \Delta \mathbf{X}_{1}+\mathbf{R}_{\mathbf{X} \omega}^{0} \phi\right)$

$c=\psi^{*}\left(\mathbf{R}_{\mathbf{X}}^{0} \phi\right)_{\mathbf{X}} \phi$

Eq. (53) is the Algebraic Branching Equation, whose solutions $\left(\beta_{1}, \beta_{2}\right)$ yield the directions tangent to bifurcating branches. Notice that, if $c=0$, one of the roots is null; this "vertical" tangent (in the sense that $\Delta \omega=0$ ) is characteristic of a pitchfork bifurcation, while the case $c \neq 0$, with two transversally intersecting branches, is a transcritical one.

\section{Conflict of Interests}

The authors declare that they have no conflict of interest.

Acknowledgements The authors would like to acknowledge the financial support of CEA Saclay. 


\section{References}

1. Y.A. Kuznetsov, Elements of Applied Bifurcation Theory. Springer New York (2004)

2. E. Doedel, B. Oldeman, Auto-07p: Continuation and bfurcation software for ordinary differential equations. Tech. rep., Concordia University (2012)

3. A. Dhooge, W. Govaerts, Y. Kuznetsov, Matcont: A matlab package for numerical bifurcation analysis of odes, SIGSAM Bull. 38(1), 21-22 (2004)

4. H. Dankowicz, F. Schilder, Recipes for Continuation. Society for Industrial and Applied Mathematics (2013)

5. L. Xie, S. Baguet, B. Prabel, R. Dufour, Bifurcation tracking by harmonic balance method for performance tuning of nonlinear dynamical systems, Mechanical Systems and Signal Processing 88, 445-461 (2017)

6. T. Detroux, L. Renson, G. Kerschen, The harmonic balance method for advanced analysis and design of nonlinear mechanical systems, in Nonlinear Dynamics, Volume 2, Springer International Publishing, pp. 19-34 (2014)

7. T. Kim, T. Rook, R. Singh, Super- and sub-harmonic response calculations for a torsional system with clearance nonlinearity using the harmonic balance method, Journal of Sound and Vibration 281(3-5), 965-993 (2005)

8. J. Inayat-Hussain, H. Kanki, N. Mureithi, On the bifurcations of a rigid response in squeeze-film dampers, Journal of Fluids and Structures 17(3), 433-459 (2003)

9. M. Krack, Nonlinear modal analysis of nonconservative systems: Extension of the periodic motion concept, Computers \& Structures 154, 59-71 (2015)

10. S. Shaw, P. Holmes, A periodically forced piecewise linear oscillator, Journal of Sound and Vibration 90(1), 129-155 (1983)

11. J. Ing, E. Pavlovskaia, M. Wiercigroch, S. Banerjee, Experimental study of impact oscillator with one-sided elastic constraint, Philosophical Transactions of the Royal Society (2008)

12. T. Detroux, G. Habib, L. Masset, G. Kerschen, Performance, robustness and sensitivity analysis of the nonlinear tuned vibration absorber, Mechanical Systems and Signal Processing 60-61, 799-809 (2015)

13. R. Kuether, L. Renson, T. Detroux, C. Grappasonni, G. Kerschen, M. Allen, Nonlinear normal modes, modal interactions and isolated resonance curves, Journal of Sound and Vibration 351, 299-310 (2016)

14. G. Habib, G. Cirillo, G. Kerschen, Uncovering detached resonance curves in single-degree-of-freedom systems, Procedia Engineering 199, 649-656 (2017)

15. G. Gatti, Uncovering inner detached resonance curves in coupled oscillators with nonlinearity, Journal of Sound and Vibration 372, 239-254 (2016)

16. C. Duan, R. Singh, Isolated sub-harmonic resonance branch in the frequency response of an oscillator with slight asymmetry in the clearance, Journal of Sound and Vibration 314(1-2), 12-18 (2008)

17. E. de Langre, G. Lebreton, An experimental and numerical analysis of chaotic motion in vibration with impact, in Flow-Induced Vibration, vol. 328, ed. by M. Pettigrew. American Society of Mechanical Engineers, vol. 328, pp. 317-325 (1996)

18. T. Cameron, J. Griffin, An alternating frequenct/time domain method for calculating the steady-state response of nonlinear dynamic systems, Journal of Applied Mechanics, ASME 56(1), 149-154 (1989)

19. T. Detroux, L. Renson, L. Masset, G. Kerschen, The harmonic balance method for bifurcation analysis of largescale nonlinear mechanical systems, Computer Methods in Applied Mechanics and Engineering 296, 18-38 (2015)

20. W.J. Kim, N. Perkins, Harmonic balance/galerkin method for non-smooth dynamic systems, Journal of Sound and Vibration 261(2), 213-224 (2003)

21. J. van Til, F. Alijani, S. Voormeeren, W. Lacarbonara, Frequency domain modeling of nonlinear end stop behavior in tuned mass damper systems under single- and multi-harmonic excitations, Journal of Sound and Vibration 438, 139-152 (2018)

22. T. Kim, T. Rook, R. Singh, Effect of smoothening functions on the frequency response of an oscillator with clearance non-linearity, Journal of Sound and Vibration 263(3), 665-678 (2003)

23. J.Y. Yoon, B. Kim, Effect and feasibility analysis of the smoothening functions for clearance-type nonlinearity in a practical driveline system, Nonlinear Dynamics 85(3), 1651-1664 (2016)

24. E. Allgower, K. Georg, Numerical Continuation Methods. Springer Berlin Heidelberg (1990)

25. B. Cochelin, C. Vergez, A high order purely frequencybased harmonic balance formulation for continuation of periodic solutions, Journal of Sound and Vibration 324, 243-262 (2009)

26. G.V. Groll, D. Ewins, The harmonic balance method with arc-length continuation in rotor/stator contact problems, Journal of Sound and Vibration 241(2), 223-233 (2001)

27. B. Bentvelsen, A. Lazarus, Modal and stability analysis of structures in periodic elastic states: application to the ziegler column, Nonlinear Dynamics 91(2), 1349-1370 (2017)

28. R. Seydel, Practical Bifurcation and Stability Analysis. Springer (2010)

29. H. Keller, Numerical solution of bifurcation and nonlinear eigenvalue problems, in Applications of Bifurcation Theory, ed. by P. Rabinowitz. Academic Press, pp. 395384 (1977) 
30. A. Nayfeh, B. Balachandran, Applied Nonlinear Dynamics. John Wiley \& Sons (2004)

31. A. Grolet, F. Thouverez, On a new harmonic selection technique for harmonic balance method, Mechanical Systems and Signal Processing 30, 43-60 (2012)

32. V.D. Witte, F.D. Rossa, W. Govaerts, Y. Kuznetsov, Numerical periodic normalization for codim 2 bifurcations of limit cycles: Computational formulas, numerical implementation, and examples, SIAM Journal on Applied Dynamical Systems 12(2), 722-788 (2013)

33. D. Dellwo, H. Keller, B. Matkowsky, E. Reiss, On the birth of isolas, SIAM Journal on Applied Mathematics 42(5), 956-963 (1982)

34. J. Kernevez, Y. Liu, M. Seoane, E. Doedel, Optimization by continuation, in Continuation and Bifurcations: $\mathrm{Nu}$ merical Techniques and Applications, Springer Netherlands, pp. 349-362 (1990)

35. S. Natsiavas, H. Gonzalez, Vibration of harmonically excited oscillators with asymmetric constraints, Journal of Applied Mechanics 59(2S), S284 (1992)

36. R. Leine, D. van Campen, B. van de Vrande, Bifurcations in nonlinear discontinuous systems, Nonlinear Dynamics 23(2), 105-164 (2000)

37. A. Ferri, M. Leamy, Error estimates for harmonicbalance solutions of nonlinear dynamical systems, in 50th AIAA/ASME/ASCE/AHS/ASC Structures, Structural Dynamics, and Materials Conference. American Institute of Aeronautics and Astronautics (2009)

38. S. Strogatz, Nonlinear Dynamics and Chaos : with Applications to Physics, Biology, Chemistry, and Engineering. Westview Press (2015) 